\title{
A cross-cultural comparison of folk plant uses among Albanians, Bosniaks, Gorani and Turks living in south Kosovo
}

\author{
Behxhet Mustafa ${ }^{1}$, Avni Hajdari ${ }^{*}$, Andrea Pieroni ${ }^{2}$, Bledar Pulaj ${ }^{1}$, Xhemaji Koro $^{1}$ and Cassandra L Quave ${ }^{3,4}$
}

\begin{abstract}
Background: Kosovo represents a unique hotspot of biological and cultural diversity in Europe, which allows for interesting cross-cultural ethnobotanical studies. The aims of this study were twofold: 1) to document the state of traditional knowledge related to local (esp. wild) plant uses for food, medicine, and handicrafts in south Kosovo; and 2) to examine how communities of different ethnic groups in the region (Albanians, Bosniaks/Gorani, and Turks) relate to and value wild botanical taxa in their ecosystem.

Methods: Field research was conducted in 10 villages belonging to the Prizren municipality and 4 villages belonging to the Dragash municipality, located in the Sharr Mountains in the southern part of Kosovo. Snowball sampling techniques were used to recruit 139 elderly informants (61 Albanians, 32 Bosniaks/Gorani and 46 Turks), for participation in semi-structured interviews regarding the use of the local flora for medicinal, food, and handicraft purposes.

Results: Overall, we recorded the local uses of 114 species were used for medicinal purposes, 29 for food (wild food plants), and 20 in handicraft activities. The most important species used for medicinal purposes were Achillea millefolium L., Sambucus nigra L., Urtica dioica L., Tilia platyphyllos Scop. Hypericum perforatum L., Chamomilla recutita (L.) Rauschert, Thymus serpyllum L. and Vaccinium myrtillus L. Chamomilla recutita was the most highly valued of these species across the populations surveyed. Out of 114 taxa used for medicinal purposes, only 44 species are also included in the European Pharmacopoeia. The predominantly quoted botanical families were Rosaceae, Asteraceae, and Lamiaceae. Comparison of the data recorded among the Albanian, Bosniak/Gorani, and Turkish communities indicated a less herbophilic attitude of the Albanian populations, while most quoted taxa were quoted by all three communities, thus suggesting a hybrid character of the Kosovar plant knowledge.
\end{abstract}

Conclusion: Cross-cultural ethnobiological studies are crucial in the Balkans not only for proposing ways of using plant natural resources, which could be exploited in sustainable local development projects (e.g. focusing on eco-tourism and small-scale trade of medicinal herbs, food niche and handicrafts products), but also for fostering collaboration and reconciliation among diverse ethnic and religious communities.

Keywords: Ethnobotany, Sharr Mountains, Folk medicine, Kosovo, Medicinal plants, Wild food plants

\footnotetext{
* Correspondence: avni.hajdari@uni-pr.edu

${ }^{1}$ Institute of Biological and Environmental Research, University of Prishtina

"Hasan Prishtina", Mother Teresa, 1000 Prishtinë, Kosovo

Full list of author information is available at the end of the article
} 


\section{Introduction}

Over the last decade, the Western Balkans have become the arena of a remarkable number of ethnobiological field studies, which have focused on territories of Bosnia and Herzegovina [1-7], Serbia [8-12], Montenegro [13], Albania [14-19], Macedonia [20-24], and Kosovo [25,26]. Moreover, a few of these studies addressed cross-cultural comparisons in an attempt to try to understand cultural concepts underpinning perceptions and uses of specific plants, especially among Albanian vs. Slavic populations $[10,15,21]$. Much of this focus on Balkan ethnobotany is linked to the long and ongoing history of gathering and trading local wild medicinal plants from this territory into Western European markets. It is also supported by the growing appreciation of ethnobotanical bio-cultural heritage as a starting point for fostering a peaceful and sustainable development in the area.

As part of our ongoing long-term project of documenting the ethnobotanical knowledge of diverse multicultural and religious areas in the Balkans, here we focused our attention on the Prizren and Dragash municipalities (South Kosovo), where traditionally diverse ethnic groups (Albanians, Turks, Bosniaks, Serbians, Gorani, Roma/Gypsies, Egyptians and Ashkali) have lived in close contact for many centuries. Previous ethnobotanical and ethnolinguistic studies conducted in Kosovo have demonstrated that medicinal plants still play a crucial role in the sphere of human health, especially in isolated rural areas [25-27]. Oftentimes, these mountainous communities have limited access to Western biomedical facilities, and they rely heavily on traditional ecological knowledge (TEK) to meet their dietary and medical needs. It is for this reason that we project that investigation of Kosovo's diverse ethnobotanical heritage will have a tremendous impact on rural development projects aimed at improving the holistic and long-term well-being of the local populations via sustainable use of local natural resources and integration of emic concepts of health and dietary care into development plans.

The aims of this study were twofold: 1) to document the state of traditional knowledge related to local (esp. wild) plant uses for food, medicine, and handicrafts in southwest Kosovo; and 2) to examine how communities of different ethnic groups in the region (Albanians, Bosniaks/Gorani, and Turks) relate to and value wild botanical taxa in their ecosystem.

\section{Methods}

\section{The study area}

In this study, we investigated traditional ecological knowledge (TEK) concerning the use of local plants in villages situated in the territory of Prizren, which lies in the southwestern part of the Sharr Mountains (in Albanian known as Malet e Sharrit; in Serbo-Croatian as Šar Planina).
The Sharr Mountains lie in the Republic of Macedonia and Kosovo and have a total area of 1,600 $\mathrm{km}^{2}$. The Republic of Macedonia is home to $51 \%\left(827 \mathrm{~km}^{2}\right)$ of this mountain range, while the Republic of Kosovo is home to the rest $\left(780 \mathrm{~km}^{2}\right)$ [28]. The Sharr Mountains provide an interesting site of plant life richness and diversity, with an estimated 2,000 vascular plant species. Indeed, a special characteristic of the Sharr Mountains is the presence of endemic, relict, and rare species and plant communities [29]. The most representative vegetation includes black alder communities (Alnetum glutinosae), which is widespread along the streams and rivers, oriental hornbeam forest (Carpinetum orientalis scardicu), hop hornbeam mixed with oriental hornbeam forest (Ostryo-Carpinion orientalis), thermophilous oak forests (Quercetum frainetto-cerris scardicum, and Quercetum pubenscens, Quercetum montanum, Quercetum trojanae dukagjini), beech forests (Fagetum montanum), and pine forests (Pinetum heldreichii, Pinetum peucis, Pinetum mughi typicum) [30].

In recognition of the rich levels of biodiversity in this region, in 1986 the Kosovo Assembly (former Autonomous Province of Kosovo within the Socialist Federal Republic of Yugoslav) declared that a part of the Sharr Mountains would be a National Park with the size of around 30,000 hectares. In 2012, the borders of the National Park were expanded and at the same time the massif of Koritnik was included, increasing the park's territory by around 23,469 hectares. Now recognized as the Sharr National Park (Figure 1), it occupies 53,469 hectares, and includes the territories of five municipalities: Kaçanik, Shtërpcë, Suharekë, Prizren and Dragash [31].

Over the past two millennia, this region has been continuously occupied and was part of three great empires (Roman, Byzantine and Ottoman). In the intervals between the decline of one emperor and empowerment of another, Kosovo was occupied mainly by Bulgarians and Serbs. According to Schmitt [32], when the Romans

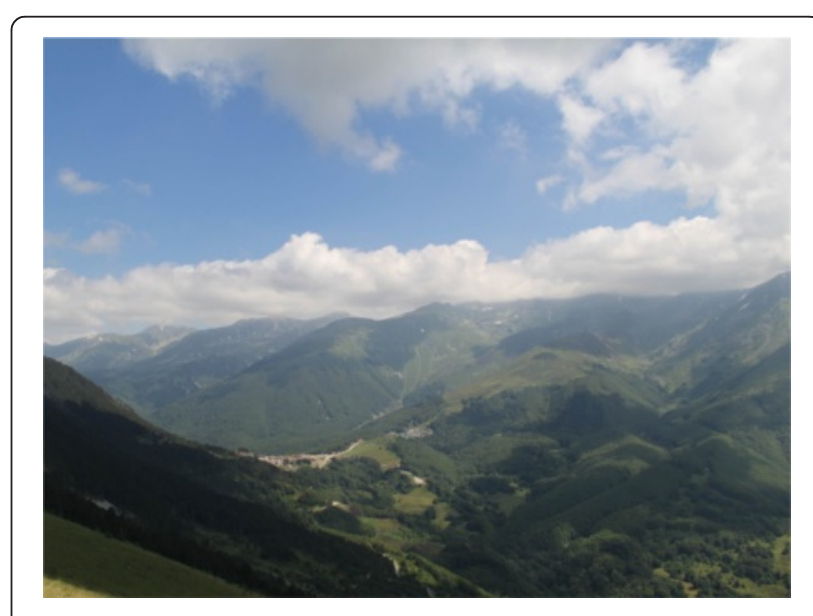

Figure 1 Landscape of the Sharr National Park. 
arrived in the Kosovar territory, they were faced with various Illyrian tribes. In late antiquity, the Dardanians became mainly Christian (Catholic); Byzantine and Slavic invasions led to the Catholicism of a significant proportion of indigenous Albanian population, despite a great resistance to the acceptance of Orthodox religion. The later Ottoman occupation spanning about five centuries resulted in conversion to Islam as the dominant religion. Because of this complex history, today the territory surrounding Prizren is occupied by diverse ethnic groups (Albanians, Serbs, Turks, Bosniaks, Gorani and Romani) and diverse religions (Muslim (Albanians, Turks, Bosniaks and Gorani), Catholic (Albanians) and Orthodox (Serbians)). The intercultural mixing of various communities in the same area has resulted in a dynamic form of TEK, with the impact of one traditional culture on another illustrated in both the uses and names of useful plants found in the local flora.

Before World War II, healthcare in this region was almost entirely based on traditional medicine, and these traditions continued after the war as well. Healthcare was commonly attended to within the family, and all physical and mental illnesses were treated with traditional medicines and rituals. These folk-medical traditions continue even now, especially in the more mountainous and isolated areas. Local people have withstood the extreme conditions of this region for centuries - including very harsh winters. Until very recent decades, limitations in infrastructure and communication forced local residents to be self-sufficient in the provision of their food and healthcare. As a result, their primary pharmacopoeia consisted of local medicinal plants.

Today, the residents southwest Kosovo are ethnic Albanians (who speak Gheg varieties of the Albanian language), Serbians (Serbian language), Turks (Turkish language), Bosniaks (Bosnian language), Gorani (Slavic language, Gora dialect or "Našinski" which is similar to Bosnian language) and Roma (Romani language). Regarding the population census conducted in 2011, there were 177,781 inhabitants in the Prizren municipality (145,718 Albanians, 237 Serbians, 9,091 Turks, 16,896 Bosniaks, 2,899 Roma, 1,350 Ashkali, 168 Egyptians, 655 Gorani and 386 others) and 33,997 in the Dragash municipality (20,287 Albanians, 7 Serbians, 202 Turks, 4,100 Bosniaks, 3 Roma, 4 Ashkali, 3 Egyptians, 8,957 Gorani, and 283 others) [33]. Population numbers and the ethnic structure of these municipalities have fluctuated over time due to the natural growth and the migration of the population. Most recently, local populations have been negatively affected by migration due to displacement and the harsh economic conditions caused by the last Kosovo War (1998-1999). The most common directions of migrations in Kosovo are from rural areas to urban areas and migration abroad. Migration patterns contribute to the rapid decline of traditional knowledge of plant species used as medicine, food and handicrafts; it has also contributed to a decline the vertical transmission of oral traditional knowledge from one generation to another. Small-scale farming and pastoral activities still represent the main economic income sources for the families in the study area. This is supplemented by remittances sent by relatives living in Germany or Switzerland, where the migrations of SW Kosovo were historically directed.

\section{The field study}

Ethnobotanical field research was conducted in 14 villages belonging to the municipalities of Prizren (10 communities) and Dragash (4), located in Sharr Mountains, which are situated in the southern part of Kosovo (Figure 2). Field studies were conducted over a series of trips in 2012 and 2014. Snowball sampling methods were used to recruit informants and we particularly focused on local people who regularly use plants for medicinal purposes. Prior informed consent was obtained prior to conducting interviews and all researchers adhered to the ethical guidelines of the International Society of Ethnobiology [34].

TEK was recorded using semi-structured interviews. In particular, informal conversations were conducted around the issue of local plants traditionally used for food (wild food plants), medicine, and handicrafts. We sought in particular the following information: respondent name, age, gender, and community of residence; local botanical names of useful plants; plant part(s) used; preparation/administration details; local folk uses of plants. In total, data were collected from 139 informants: 61 Albanians (43 male, 18 female), 32 Bosniaks/Gorani (Bosniaks: 11 male, 7 female; Gorani: 10 male, 4 female) and 46 Turks (28 male, 18 female). With regards to the data analysis, data collected from the Bosniak and Gorani informants were merged as both are culturally similar and share the same religion and language. Gorani communities have been claimed by Bosniaks, Serbs, and Bulgarians and recently by Macedonians, but in Kosovo they are recognized as a distinct minority group.

The respondents were older than 50 years (with a few exceptions), mainly engaged in agricultural activities and typically inherited their ethnobotanical knowledge from their direct ancestors (parents, grandparents) via oral traditions. During the interviews, fresh plants were collected to create voucher specimens for the herbarium and whenever possible, informants were followed into the field to show us the quoted species. Most plant species were collected while flowering. Taxonomic identification was undertaken using relevant standard botanical literature of the area [35-38]. Plant nomenclature largely follows the Flora Europaea [39], while plant family assignments follow the current Angiosperm Phylogeny 


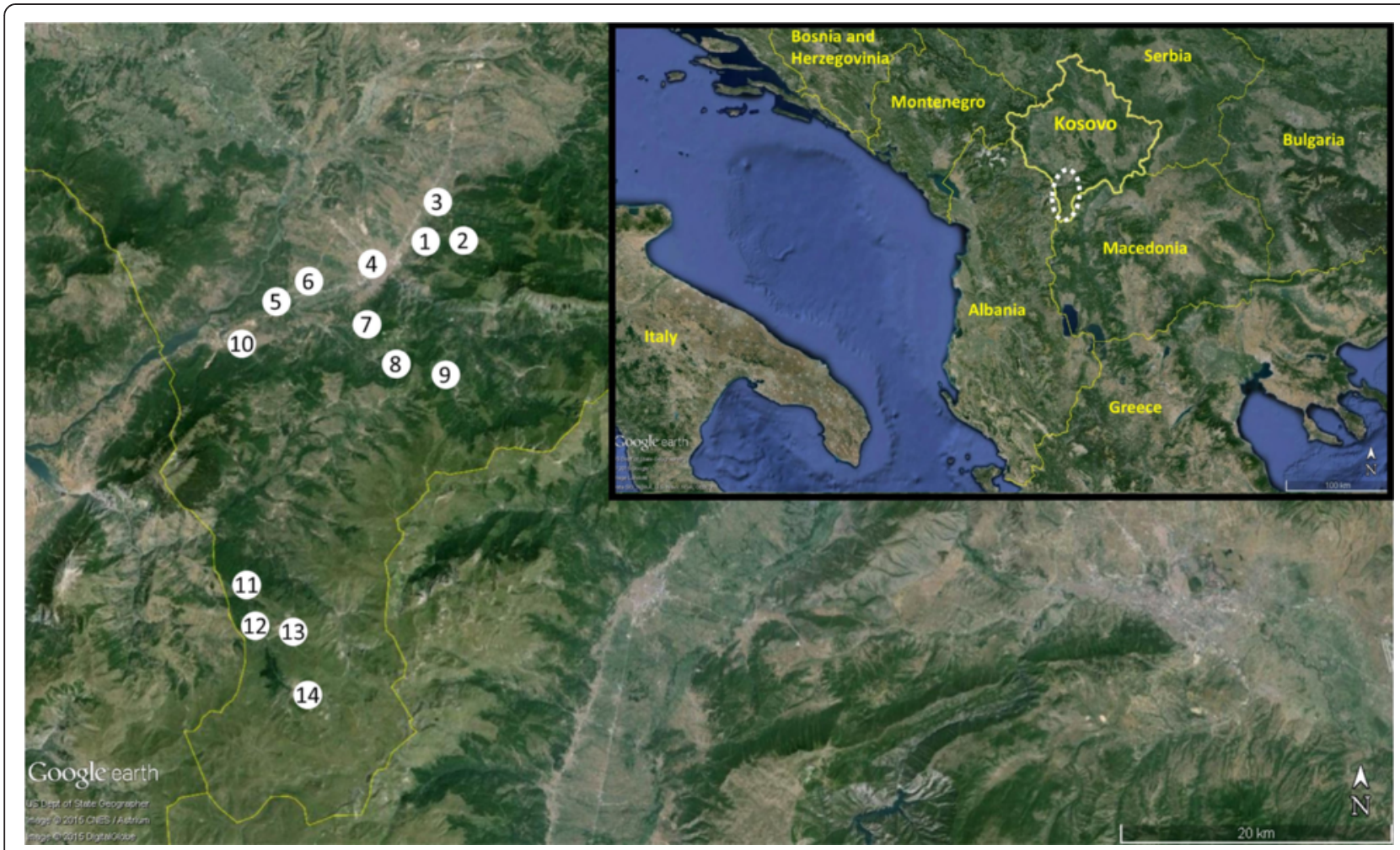

Figure 2 Map of study area and location of communities included in the study. Communities included in the study are indicated by number: 1) Gërnçar (710 m.a.s.l.), 2)Skorobisht (990 m), 3) Lubizhdë e Prizrenit (495 m), 4) Prizren (421 m), 5) Vlashnje (337 m), 6) Grazhdanik (385 m), 7) Leskovecë (830 m), 8) Lez (117 m), 9) Struzhë (1169 m), 10) Zhur (461 m), 11) Glloboçicë (1270 m), 12) Krushevë (1164 m), 13) Zlipotok (1395 m) and 14) Restelicë (1470 m).

Group III guidelines [40]. Voucher specimens of the wild taxa were deposited at the Department of Biology (Herbarium code Pz/2013), University of Prishtina.

\section{Data analysis}

\section{Overlap analysis for cited taxa}

Taxa with use-citations based on general category of use (medicinal, food or handicraft) were compared across three groups (Albanian, Turks and Bosniaks/Gorani). Data are represented in the form of a Venn Diagram (Figure 3) to illustrate overlaps in use of taxa.

\section{Use-value for individual species}

The use-value citation $\left(U V_{c}\right)$ index was calculated for each species for each ethnic group [41]. Here, we modified this method to calculate UV values in three different categories of use: medicinal, food, and handicraft. This index is useful for examination of relative importance of each species for a general category of use based on the number of use-citations. Briefly, it was calculated as follows:

$$
U V_{c}=\frac{\sum N_{u c}}{N}
$$

Where $\mathrm{N}_{\mathrm{uc}}$ is the number of use citation reports concerning a given species in a use category (e.g. medicinal, food, handicraft), divided by the total number of informants $(\mathrm{N})$ in a specific group (e.g. Albanian, Turkish, or Bosniak \& Gorani). In a recent paper by Quave and Pieroni [42], UV values were plotted on a two-dimensional matrix framework to assess relative values for individual species between two ethnic groups. Here, we expand upon this concept and apply it to a three-dimensional matrix for comparison of plant use-values for individual species between three ethnic groups that share access to the same environmental and botanical resources.

\section{Three-dimensional (3-D) use-value matrix design and analysis}

We propose a new approach for the comparative analysis of how use-values differ in three ethnic groups, and across different general categories of use. The $\mathrm{UV}_{\mathrm{c}}$ data for each category of use (medicinal, food, handicraft) 


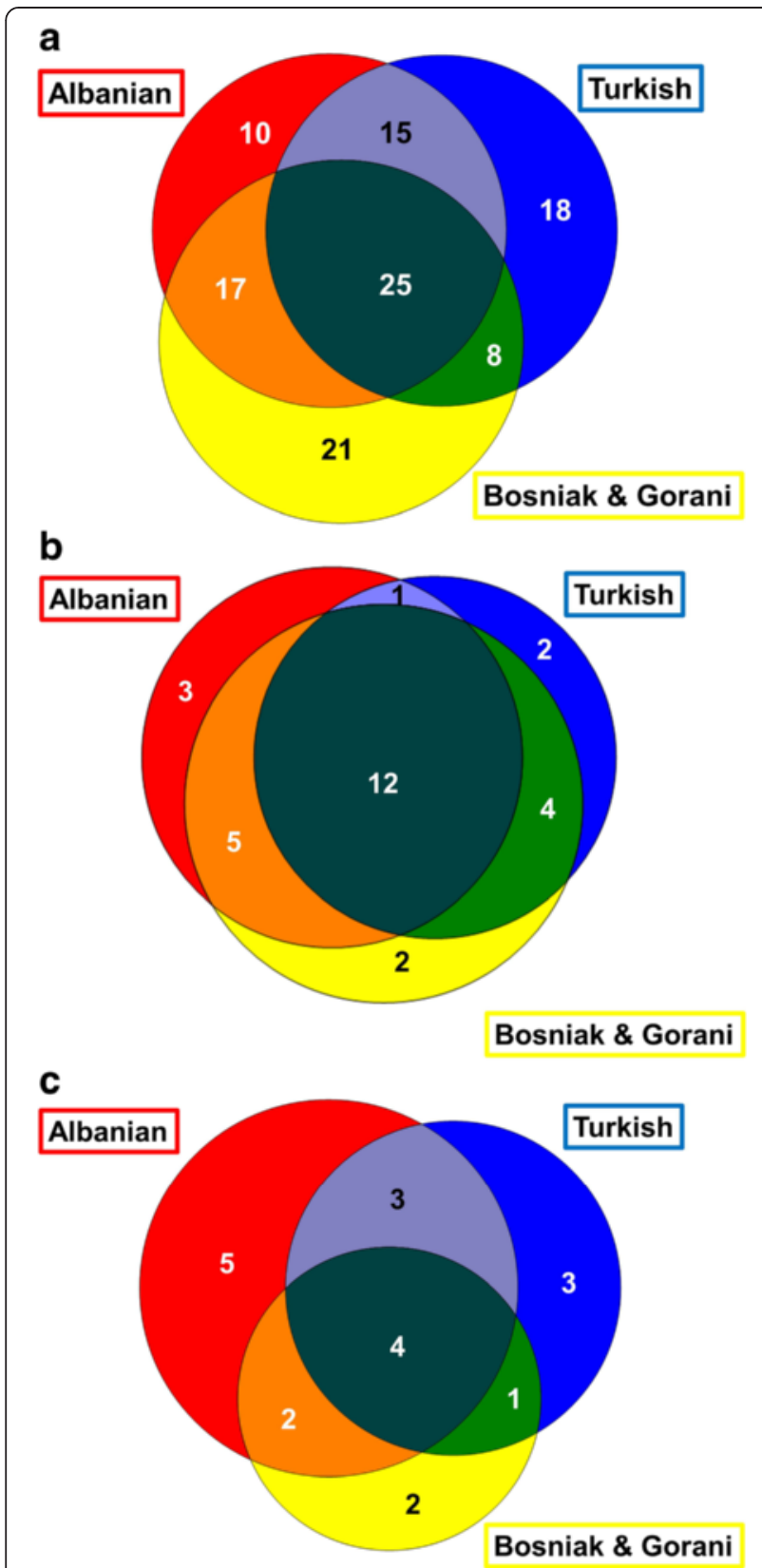

Figure 3 Venn diagram representing the overlap of taxa cited by Albanians, Bosniaks/Gorani, and Turks in the study area for: a) medicinal use, b) food use and c) handicraft use.

were normalized to allow for comparison on a scale of $0-1$. This was achieved by identifying the maximum $U V_{c}$ value for each category of use $\left(U V_{\text {max }}\right)$. The $U V_{c}$ for each species (and ethnic group) was then divided by the $\mathrm{UV}_{\text {max }}$ to create the adjusted UV value $\left(\mathrm{UV}_{\text {adj }}\right)$ and plotted onto a 3-D scatterplot using MATLAB ${ }^{\ominus}$ software. Eight 3-D overlay quadrants were created to assist in classifying the $\mathrm{UV}_{\text {adj }}$ clusters (Figure 4A). They were defined as follows in relationship to the three ethnic groups being compared (Group 1: Bosniak/Gorani; Group 2: Turkish; Group 3: Albanian):

- Quadrant I: Taxa with $U_{\text {adj }} \leq 0.05$ for all three groups, indicating consensus in low use-value across groups.

- Quadrant II: Group $1 \mathrm{UV}_{\text {adj }}>0.05$; Group $2 \mathrm{UV}_{\text {adj }}$ $\leq 0.05$; Group $3 \mathrm{UV}_{\text {adj }} \leq 0.05$, indicating consensus on lower use-value among Group 2 and 3, but higher use-value for Group 1.

- Quadrant III: Group $1 \mathrm{UV}_{\text {adj }} \leq 0.05$; Group $2 \mathrm{UV}_{\text {adj }}$ $>0.05$; Group $3 \mathrm{UV}_{\mathrm{adj}} \leq 0.05$, indicating consensus on lower use-value among Group 1 and 3, but higher use-value for Group 2.

- Quadrant IV: Group $1 \mathrm{UV}_{\text {adj }}>0.05$; Group $2 \mathrm{UV}_{\text {adj }}$ $>0.05$; Group $3 \mathrm{UV}_{\mathrm{adj}} \leq 0.05$, indicating consensus on higher use-value among Group 1 and 2, but lower use-value for Group 3.

- Quadrant V: Group $1 \mathrm{UV}_{\text {adj }} \leq 0.05$; Group $2 \mathrm{UV}_{\text {adj }}$ $\leq 0.05$; Group $3 \mathrm{UV}_{\text {adj }}>0.05$, indicating consensus on lower use-value among Group 1 and 2, but higher use-value for Group 3.

- Quadrant VI: Group $1 \mathrm{UV}_{\text {adj }}>0.05$; Group $2 \mathrm{UV}_{\text {adj }}$ $\leq 0.05$; Group $3 \mathrm{UV}_{\text {adj }}>0.05$, indicating consensus on higher use-value among Group 1 and 3, but lower use-value for Group 2.

- Quadrant VII: Group $1 \mathrm{UV}_{\text {adj }} \leq 0.05$; Group $2 \mathrm{UV}_{\text {adj }}$ $>0.05$; Group $3 U V_{\text {adj }}>0.05$, indicating consensus on higher use-value among Group 2 and 3, but lower use-value for Group 1.

- Quadrant VIII: Taxa with $U_{\text {adj }}>0.05$ for all three groups, indicating consensus in high use-value across groups.

Quadrant assignments are also reported in Tables 1, 2 and 3 .

\section{Results and discussion}

In total, TEK on the local uses of 124 taxa (belonging to 51 families) was recorded; of these, 114 species were used for medicinal purposes, 29 wild species for food, and 20 for handicrafts. Some of the cited species were used for multiple purposes. The total number of use citation $\left(\mathrm{N}_{\mathrm{uc}}\right)$ for each species is reported by ethnic group and category of use: medicinal (Table 1), food (Table 2), and handicraft (Table 3) applications.

\section{Medicinal plants}

TEK on the recorded local uses of 114 medicinal plant taxa, representing 49 taxonomic families, are reported in Table 1. Of these species, Achillea millefolium L., Sambucus nigra L., Urtica dioica L., Tilia platyphyllos Scop., Hypericum perforatum L., Matricaria chamomilla 


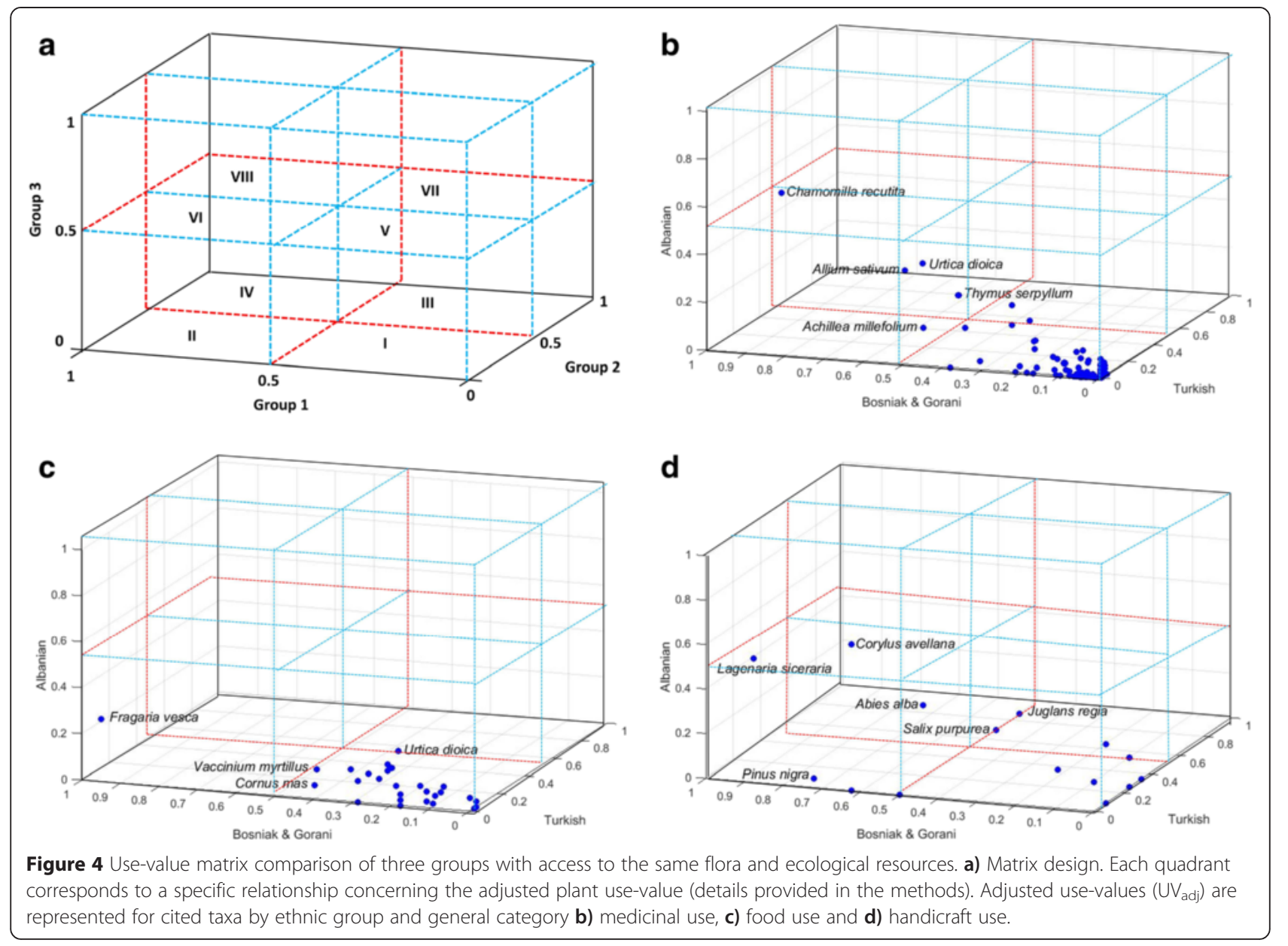

L., Thymus serpyllum L., and Vaccinium myrtillus L. were cited by more than $30 \%$ of the informants. Of the 114 cited for medicinal purposes, 44 are also included in the official Pharmacopoeia of Europe (European Pharmacopoeia. 6 ed.). The predominantly quoted botanical families were Rosaceae (13\%), Asteraceae (11\%), and Lamiaceae (10\%). These same three "top" families were found to also be predominant among the wild medicinal taxa used in the folk medicine of the Albanian Alps (Kosovo), Alps in Montenegro, Albania, and in the Gollak region of Kosovo [13,17-19,25,26].

The total number species quoted by each ethnic group were roughly equivalent: 67, 66, and 71 for the Albanians, Turks and Bosniaks, respectively. Figure 3A illustrates the overlap in citation of medicinal plant among the three populations, with 10 species used only by Albanians, 18 by Turks and 21 only by Bosniaks/Gorani. Furthermore, common uses were shared between certain groups: 15 only between Albanians and Turks, 8 only between Bosniaks/Gorani and Turks and 17 only between Albanian and Bosniaks/Gorani. A total of 25 species were cited for medicinal use by all three study populations.

The most frequently cited medicinal uses referred to gastrointestinal (17.8\%), respiratory (15.1\%) ailments, heart disease (13.6\%), illnesses affecting the urogenital system (12.4\%) and the skin (10.5\%). These categories were the most frequently quoted in the ethnobotanical studies conducted in Gollak (Kosovo) [26], while the gastrointestinal and respiratory troubles were also the most frequently quoted in the ethnobotanical studies conducted in the Albanian Alps (Kosovar, Montenegrin and Albanian sides) [13,17-19,25].

Our 3-D analysis of the data revealed that of the cited species, Chamomila recutita had the highest use-value across groups, and was assigned to Quadrant VI, demonstrating high value among Albanians and Bosniaks/ Gorani, with moderately high $\left(\mathrm{UV}_{\mathrm{adj}}=0.46\right)$ use-value among the Turkish population studied as well. While most taxa fell into Quadrant I, representing low to moderate level use-values among all three populations, two 
Table 1 Medicinal plant used in the study area

\begin{tabular}{|c|c|c|c|c|c|c|c|c|c|c|c|c|}
\hline $\begin{array}{l}\text { Botanical taxon, } \\
\text { family and voucher } \\
\text { specimen code }\end{array}$ & Status & Folk name $(s)^{a}$ & $\begin{array}{l}\text { Part(s) } \\
\text { used }\end{array}$ & Administration & $\begin{array}{l}\text { Treated disease(s) } \\
\text { or folk medical } \\
\text { uses(s) }\end{array}$ & $\begin{array}{l}\text { Alb } \\
\mathrm{N}_{\mathrm{bc}}\end{array}$ & $\begin{array}{l}\mathrm{Bo} / \\
\mathrm{Go} \\
\mathrm{N}_{\mathrm{bc}}\end{array}$ & $\begin{array}{l}\text { Tur } \\
\mathrm{N}_{\mathrm{uc}}\end{array}$ & $U_{\text {Alb }}^{c}$ & $\begin{array}{l}\mathrm{UV}_{\mathrm{Bo} /} \\
\mathrm{Go}^{\mathrm{C}}\end{array}$ & $U_{\text {Tur }}^{c}$ & $\overline{Q^{d}}$ \\
\hline $\begin{array}{l}\text { Abies alba Mill. } \\
\text { (Pinaceae) 14/Pz/2013 }\end{array}$ & W & Bredhi ${ }^{A L B}$ & Wood & $\begin{array}{l}\text { Resin, mixed } \\
\text { with fat }\end{array}$ & Anti-fungal & 2 & 0 & 0 & 0.033 & 0 & 0 & I \\
\hline \multirow{4}{*}{$\begin{array}{l}\text { Agrimonia eupatoria L. } \\
\text { (Rosaceae) 08/Pz/2013 }\end{array}$} & \multirow[t]{4}{*}{ W } & Petrovac ${ }^{\mathrm{BOG}}$ & \multirow{4}{*}{$\begin{array}{l}\text { Aerial } \\
\text { parts }\end{array}$} & \multirow[t]{4}{*}{ Infusion } & Anti-allergic, & 0 & 3 & 1 & \multirow[t]{4}{*}{0} & \multirow[t]{4}{*}{0.219} & \multirow[t]{4}{*}{0.065} & । \\
\hline & & \multirow[t]{3}{*}{ Kezell japrak ${ }^{T U R}$} & & & Earache, & 0 & 1 & 0 & & & & \\
\hline & & & & & Anti-inflammatory, & 0 & 2 & 2 & & & & \\
\hline & & & & & Anti-diarrheal & 0 & 1 & 0 & & & & \\
\hline \multirow{4}{*}{$\begin{array}{l}\text { Agropyron repens (L.) } \\
\text { Beauv. (Poaceae) 07/ } \\
\text { Pz/2013 }\end{array}$} & \multirow[t]{4}{*}{ W } & Bari i magarit ${ }^{\mathrm{ALB}}$ & \multirow{4}{*}{$\begin{array}{l}\text { Aerial } \\
\text { parts }\end{array}$} & \multirow[t]{4}{*}{ Infusion } & Anti-hemorrhoidal, & 2 & 0 & 0 & \multirow[t]{4}{*}{0.049} & \multirow[t]{4}{*}{0.125} & \multirow[t]{4}{*}{0.065} & । \\
\hline & & Priovina $^{\mathrm{ALB}}$ & & & $\begin{array}{l}\text { Respiratory system } \\
\text { disorders, }\end{array}$ & 0 & 3 & 0 & & & & \\
\hline & & \multirow{2}{*}{ Priovina $^{\mathrm{BOG}}$} & & & Urinary tract & 1 & 1 & 0 & & & & \\
\hline & & & & & disorders & & & & & & & \\
\hline $\begin{array}{l}\text { Alchemilla vulgaris L. } \\
\text { (Rosaceae) 05/Pz/2013 }\end{array}$ & $\mathrm{E}$ & Alhemila ${ }^{\text {ALB }}$ & $\begin{array}{l}\text { Aerial } \\
\text { parts }\end{array}$ & Infusion & $\begin{array}{l}\text { Improve fertility in } \\
\text { women }\end{array}$ & 2 & 0 & 0 & 0.033 & 0 & 0 & I \\
\hline \multirow{11}{*}{$\begin{array}{l}\text { Achillea millefolium L. } \\
\text { (Asteraceae) 03/ } \\
\text { Pz/2013 }\end{array}$} & \multirow[t]{11}{*}{ W } & Barëpezmatimi ${ }^{A L B}$ & \multirow[t]{11}{*}{$\begin{array}{l}\text { Aerial } \\
\text { parts }\end{array}$} & \multirow[t]{11}{*}{ Infusion } & $\begin{array}{l}\text { Anti- } \\
\text { cholesterolemic, }\end{array}$ & 0 & 4 & 0 & \multirow[t]{11}{*}{0.557} & \multirow[t]{11}{*}{2.656} & \multirow[t]{11}{*}{0.652} & । \\
\hline & & $\begin{array}{l}\text { Hajdutska } \\
\text { trava }^{B O G}\end{array}$ & & & Anti-coagulant, & 3 & 6 & 0 & & & & \\
\hline & & \multirow[t]{9}{*}{ Hajdut oti ${ }^{\text {TUR }}$} & & & Appetizing, & 5 & 3 & 6 & & & & \\
\hline & & & & & Anti-microbial, & 12 & 28 & 8 & & & & \\
\hline & & & & & Antiemetic, & 2 & 0 & 0 & & & & \\
\hline & & & & & $\begin{array}{l}\text { Carminative and } \\
\text { spasmolytic, }\end{array}$ & 7 & 31 & 11 & & & & \\
\hline & & & & & Anti-diabetic, & 0 & 4 & 1 & & & & \\
\hline & & & & & Antacid, & 0 & 1 & 0 & & & & \\
\hline & & & & & Menstrual pains, & 0 & 1 & 0 & & & & \\
\hline & & & & & Influenza, & 2 & 5 & 3 & & & & \\
\hline & & & & & Stomachache & 3 & 2 & 1 & & & & \\
\hline & C & Kepa ${ }^{\mathrm{ALB}}$ & Bulbs & Eaten raw, & Anti-cholesterolemic & 3 & 7 & 1 & 0.656 & 1.125 & 0.5 & । \\
\hline $\begin{array}{l}\text { (Amaryllidaceae) 11/ } \\
\text { Pz/2013 }\end{array}$ & & Cerveni luk ${ }^{B O G}$ & & Topically in & Anti-bacterial & 37 & 29 & 22 & & & & \\
\hline & & Kepi ${ }^{\mathrm{TUR}}$ & & & & & & & & & & \\
\hline Allium porrum L. & C & Purri ${ }^{A L B}$ & Leaves & Eaten & Thyroid disorders & 2 & 4 & 0 & 0.033 & 0.125 & 0 & । \\
\hline $\begin{array}{l}\text { (Amaryllidaceae) 09/ } \\
\text { Pz/2013 }\end{array}$ & & Prazilluk ${ }^{\mathrm{BOG}}$ & & & & & & & & & & \\
\hline Allium sativum $\mathrm{L}$. & C & Hudra $\mathrm{ALB}$ & Bulbs & Eaten & Anti-hypertensive, & 16 & 24 & 11 & 1.361 & 3.188 & 1.63 & $\|$ \\
\hline $\begin{array}{l}\text { (Amaryllidaceae) 10/ } \\
\text { Pz/2013 }\end{array}$ & & Beli lluk ${ }^{B O G}$ & & & Anti-fungal, & 11 & 8 & 3 & & & & \\
\hline & & $\mathrm{Sa}$ & & & Anti-ageing, & 0 & 3 & 5 & & & & \\
\hline & & & & & $\begin{array}{l}\text { Urinary tract } \\
\text { infections, }\end{array}$ & 2 & 5 & 3 & & & & \\
\hline & & & & & Anti-hypertensive, & 18 & 14 & 12 & & & & \\
\hline & & & & & Bronchitis & 12 & 6 & 8 & & & & \\
\hline & & & & Mixed with & Bronchitis, & 14 & 7 & 7 & & & & \\
\hline & & & & & Anti-tussive, & 2 & 8 & 3 & & & & \\
\hline & & & & & $\begin{array}{l}\text { Skeletal system } \\
\text { enhancement, }\end{array}$ & 0 & 2 & 2 & & & & \\
\hline & & & & & Immunostimulant, & 0 & 5 & 3 & & & & \\
\hline
\end{tabular}


Table 1 Medicinal plant used in the study area (Continued)

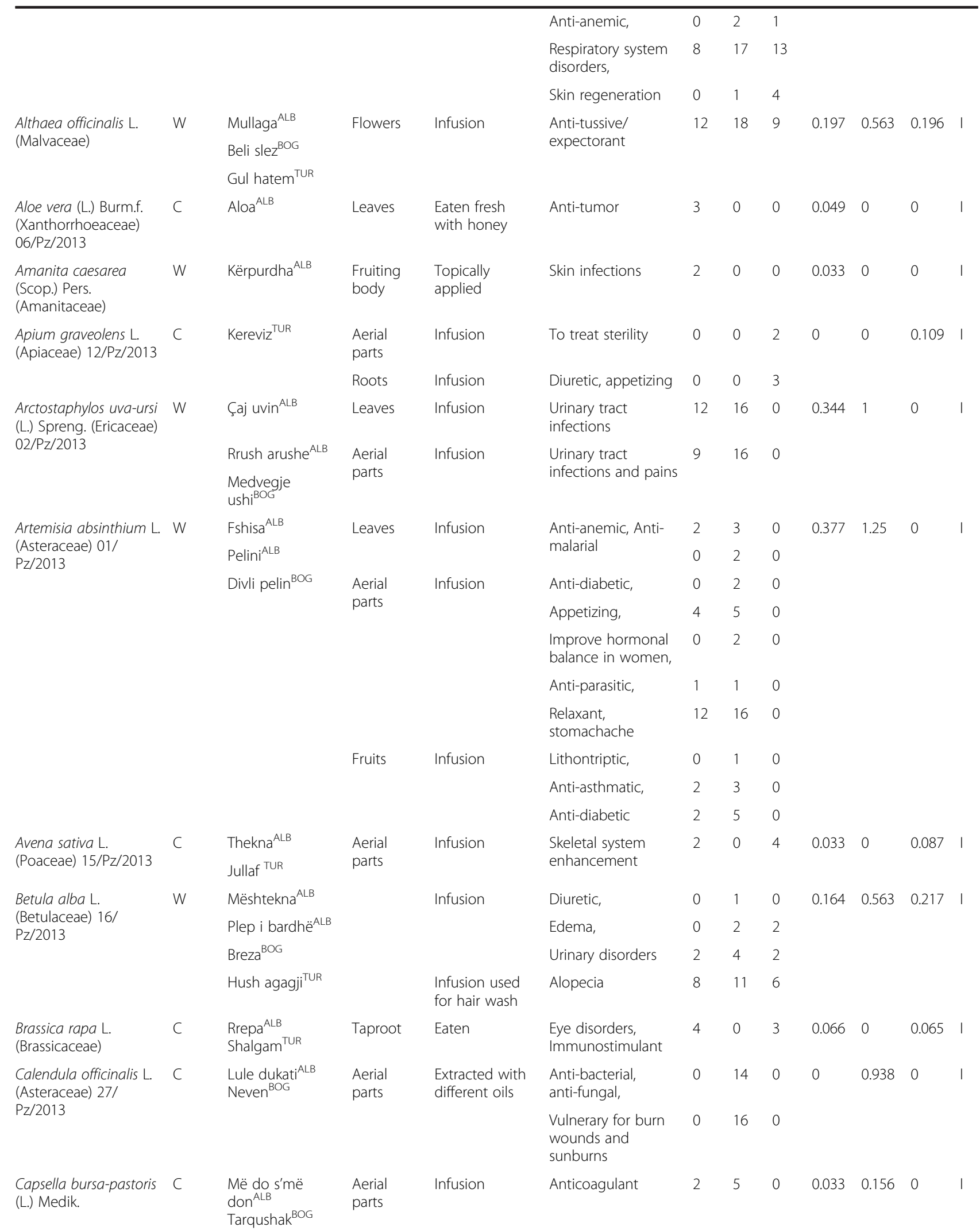


Table 1 Medicinal plant used in the study area (Continued)

\begin{tabular}{|c|c|c|c|c|c|c|c|c|c|c|c|c|}
\hline $\begin{array}{l}\text { (Brassicaceae) 28/ } \\
\text { Pz/2013 }\end{array}$ & & Hoqunequ $^{\mathrm{BOG}}$ & & & & & & & & & & \\
\hline \multirow{4}{*}{$\begin{array}{l}\text { Castanea sativa Mill. } \\
\text { (Fagaceae) 19/Pz/2013 }\end{array}$} & \multirow[t]{4}{*}{ W/C } & \multirow[t]{4}{*}{ Gështaja ${ }^{A L B}$} & \multirow[t]{3}{*}{ Flowers } & \multirow[t]{3}{*}{ Infusion } & Anti-anemic, & 3 & 0 & 0 & \multirow[t]{4}{*}{0.131} & \multirow[t]{4}{*}{0} & \multirow[t]{4}{*}{0} & \multirow[t]{4}{*}{ । } \\
\hline & & & & & Bronchitis, & 2 & 0 & 0 & & & & \\
\hline & & & & & Anti-tussive & 2 & 0 & 0 & & & & \\
\hline & & & Cortex & Infusion & Anti-tussive & 1 & 0 & 0 & & & & \\
\hline \multirow{7}{*}{$\begin{array}{l}\text { Centaurium erythraea } \\
\text { Rafn (Gentianaceae) } \\
\text { 29/Pz/2013 }\end{array}$} & \multirow[t]{7}{*}{ W } & $\begin{array}{l}\text { Kantarioni i } \\
\text { kuq }^{\text {ALB }}\end{array}$ & \multirow[t]{7}{*}{$\begin{array}{l}\text { Aerial } \\
\text { parts }\end{array}$} & \multirow[t]{7}{*}{ Infusion } & Anticoagulant, & 0 & 2 & 0 & \multirow[t]{7}{*}{0.426} & \multirow[t]{7}{*}{1.063} & \multirow[t]{7}{*}{0.391} & । \\
\hline & & Bari i etheve ${ }^{A L B}$ & & & Anti-pyretic, & 13 & 18 & 9 & & & & \\
\hline & & \multirow[t]{5}{*}{ Kicica ${ }^{\mathrm{BOG}}$} & & & Anti-malarial, & 9 & 12 & 6 & & & & \\
\hline & & & & & Appetizing, & 2 & 1 & 0 & & & & \\
\hline & & & & & Anti-anemic, & 0 & 1 & 0 & & & & \\
\hline & & & & & Antacid, & 0 & 0 & 1 & & & & \\
\hline & & & & & Immunostimulant & 2 & 0 & 2 & & & & \\
\hline \multirow{2}{*}{$\begin{array}{l}\text { Centaurea cyanus L. } \\
\text { (Asteraceae) 20/ } \\
\text { Pz/2013 }\end{array}$} & \multirow[t]{2}{*}{ W } & KokoçeliALB & \multirow[t]{2}{*}{ Flower } & \multirow[t]{2}{*}{ Infusion } & \multirow{2}{*}{$\begin{array}{l}\text { Respiratory } \\
\text { disorders }\end{array}$} & \multirow[t]{2}{*}{3} & 1 & 0 & 0.049 & 0.031 & 0 & I \\
\hline & & Kicica ${ }^{\mathrm{BOG}}$ & & & & & & & & & & \\
\hline Chamomilla recutita & W & Lule qeni ${ }^{A L B}$ & Aerial & Infusion & Antimicrobial, & 31 & 37 & 25 & 3.115 & 5.5 & 2.543 & $\mathrm{VI}$ \\
\hline $\begin{array}{l}\text { (L.) Rauschert } \\
\text { Pz/2013 }\end{array}$ & & Kamomil $^{\mathrm{ALB}}$ & & & $\begin{array}{l}\text { Infections of the } \\
\text { digestive tract, }\end{array}$ & 12 & 10 & 0 & & & & \\
\hline & & Kamilica $^{\mathrm{BOG}}$ & & & $\begin{array}{l}\text { Urinary tract } \\
\text { infections, }\end{array}$ & 20 & 6 & 13 & & & & \\
\hline & & & & & Eye infections & 11 & 8 & 2 & & & & \\
\hline & & Babunec $^{\mathrm{BOG}}$ & & & & 25 & 15 & 17 & & & & \\
\hline & & Papatja $^{\text {TUR }}$ & & & & & & & & & & \\
\hline & & Sari çiçek ${ }^{T U R}$ & & & & & & & & & & \\
\hline & & & Flowers & Infusion & Anti-tussive, & 14 & 26 & 22 & & & & \\
\hline & & & & & Anti-bacterial, & 31 & 35 & 12 & & & & \\
\hline & & & & & Influenza, & 11 & 9 & 9 & & & & \\
\hline & & & & & $\begin{array}{l}\text { Oral cavity } \\
\text { infections, }\end{array}$ & 9 & 3 & 4 & & & & \\
\hline & & & & & Anti-hemorrhoidal, & 1 & 0 & 7 & & & & \\
\hline & & & & & Alopecia, & 2 & 0 & 0 & & & & \\
\hline & & & & & Wound healing, & 20 & 23 & 6 & & & & \\
\hline & & & & & Relaxant & 3 & 4 & 0 & & & & \\
\hline Chelidonium majus $\mathrm{L}$. & W & Tamelqak ${ }^{\text {ALB }}$ & Latex & Topically used & Skin infections, & 11 & 0 & 7 & 0.18 & 0 & 0.152 & । \\
\hline $\begin{array}{l}\text { (Papaveraceae) 30/ } \\
\text { Pz/2013 }\end{array}$ & & Kena qıqegı ${ }^{\text {TUR }}$ & & & warts & & & & & & & \\
\hline Cichorium intybus $\mathrm{L}$. & W & Cikorja ${ }^{\mathrm{SHQ}}$ & Aerial & Infusion & Hepatic disorders & 2 & 0 & 4 & 0.033 & 0 & 0.087 & । \\
\hline $\begin{array}{l}\text { (Asteraceae) 21/ } \\
\text { Pz/2013 }\end{array}$ & & Mavi çiçek ${ }^{T U R}$ & parts & & & & & & & & & \\
\hline & & Satali bitki TUR & & & & & & & & & & \\
\hline Citrus limon (L.) & C & $\operatorname{Limun}^{\mathrm{BOG}}$ & Fruits & Lemon juice & Anti-tussive, & 0 & 6 & 3 & 0 & 0.188 & 0.065 & । \\
\hline $\begin{array}{l}\text { Osbeck (Rutaceae) 31/ } \\
\text { Pz/2013 }\end{array}$ & & $\operatorname{Limon}^{T U R}$ & & $\begin{array}{l}\text { mixed with } \\
\text { sugar }\end{array}$ & bronchitis & & & & & & & \\
\hline Cornus mas L. & W & Thana ${ }^{A L B}$ & Fruits & Infusion & Anti-anemic, & 0 & 5 & 1 & 0.148 & 0.656 & 0.304 & । \\
\hline $\begin{array}{l}\text { (Cornaceae) 23/ } \\
\text { Pz/2013 }\end{array}$ & & Drenilje ${ }^{B O G}$ & & & Anti-hypertensive, & 5 & 3 & 6 & & & & \\
\hline & & Dirnina $^{\text {TUR }}$ & & & Anti-diarrheal, & 0 & 4 & 2 & & & & \\
\hline & & & & & Anti-malarial, & & 1 & 1 & & & & \\
\hline
\end{tabular}




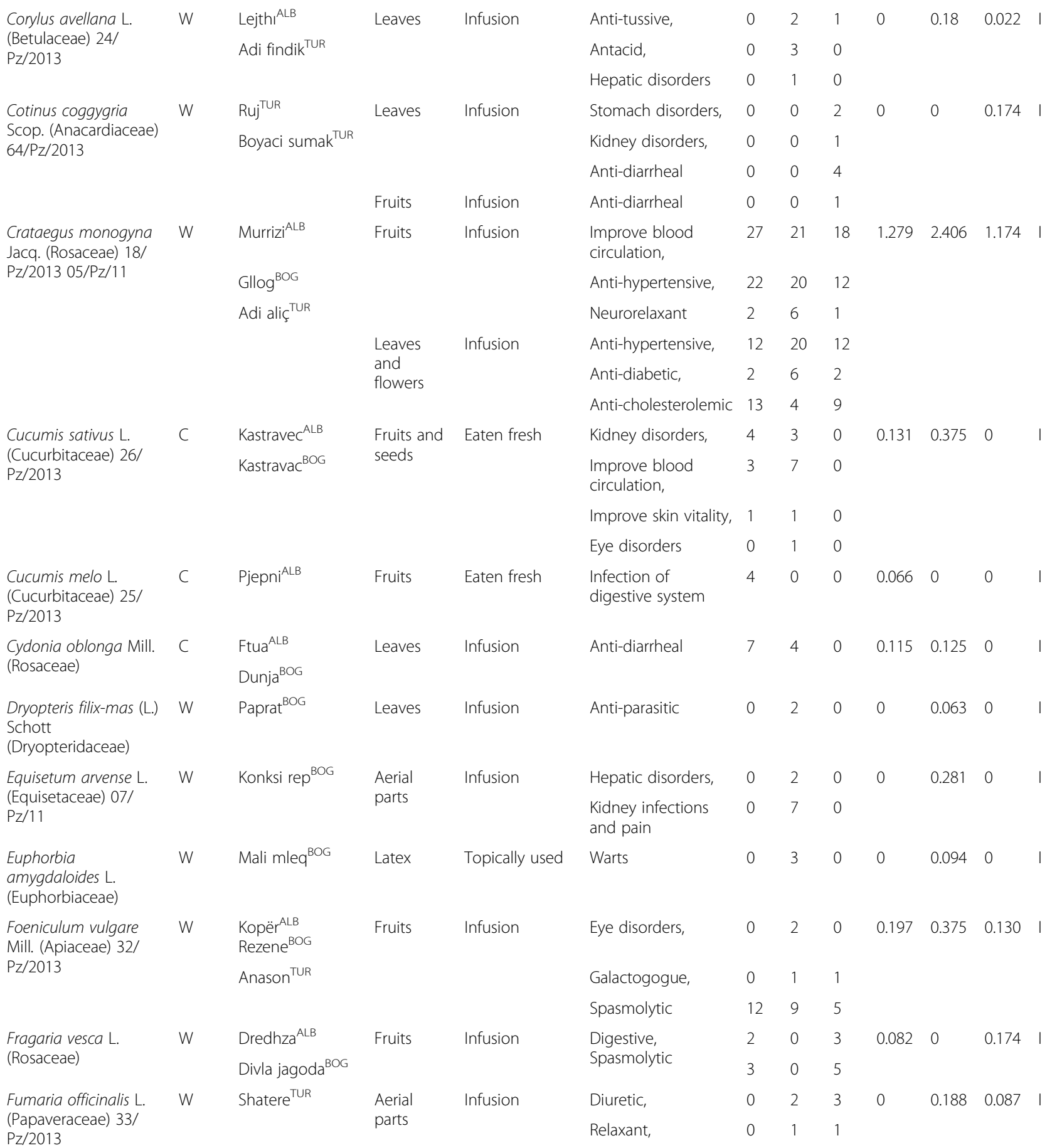


Table 1 Medicinal plant used in the study area (Continued)

\begin{tabular}{|c|c|c|c|c|c|c|c|c|c|c|c|c|}
\hline & & & & & Anti-hypertensive & 0 & 3 & 0 & & & & \\
\hline \multirow{2}{*}{$\begin{array}{l}\text { Galium verum L. } \\
\text { (Rubiaceae) 35/ } \\
\text { Pz/2013 }\end{array}$} & \multirow[t]{2}{*}{ W } & \multirow[t]{2}{*}{ / } & \multirow{2}{*}{$\begin{array}{l}\text { Aerial } \\
\text { parts }\end{array}$} & \multirow[t]{2}{*}{ Infusion } & Kidney disorders, & 0 & 0 & 2 & \multirow[t]{2}{*}{0} & \multirow[t]{2}{*}{0} & \multirow[t]{2}{*}{0.130} & \multirow[t]{2}{*}{ I } \\
\hline & & & & & Skin regeneration & 0 & 0 & 4 & & & & \\
\hline \multirow{2}{*}{$\begin{array}{l}\text { Gentiana lutea L. } \\
\text { (Gentianaceae) 34/ } \\
\text { Pz/2013 }\end{array}$} & \multirow[t]{2}{*}{ W } & Geciana $^{\mathrm{ALB}}$ & \multirow[t]{2}{*}{ Roots } & \multirow[t]{2}{*}{ Infusion } & Digestive disorders, & 5 & 12 & 0 & \multirow[t]{2}{*}{0.180} & \multirow[t]{2}{*}{0.375} & \multirow[t]{2}{*}{0} & \multirow[t]{2}{*}{ I } \\
\hline & & Lincura $^{\mathrm{BOG}}$ & & & $\begin{array}{l}\text { Flavor additive for } \\
\text { alcoholic beverage }\end{array}$ & 6 & 0 & 0 & & & & \\
\hline $\begin{array}{l}\text { Geranium sanguineum } \\
\text { L. (Geraniaceae) }\end{array}$ & W & Zdrvac ${ }^{\mathrm{BOG}}$ & $\begin{array}{l}\text { Aerial } \\
\text { parts }\end{array}$ & Infusion & $\begin{array}{l}\text { Respiratory } \\
\text { disorders, laryngitis }\end{array}$ & 0 & 3 & 0 & 0 & 0.094 & 0 & 1 \\
\hline \multirow{2}{*}{$\begin{array}{l}\text { Helianthus annuus L. } \\
\text { (Asteraceae) }\end{array}$} & \multirow[t]{2}{*}{ C } & Lule djellıALB & \multirow[t]{2}{*}{ Seeds } & \multirow{2}{*}{$\begin{array}{l}\text { Extracted with } \\
\text { animal fat }\end{array}$} & \multirow[t]{2}{*}{ Skin infections } & \multirow[t]{2}{*}{6} & 5 & 0 & 0.098 & 0.156 & 0 & 1 \\
\hline & & Suncokret ${ }^{\mathrm{BOG}}$ & & & & & & & & & & \\
\hline Hordeum vulgare Jess. & $C$ & $E l b i^{A L B}$ & Seeds & Flour, mixed & Wound healing & 5 & 0 & 2 & 0.082 & 0 & 0.043 & l \\
\hline (Poaceae) & & Jeçmenik ${ }^{T U R}$ & & with oil & & & & & & & & \\
\hline & & Arpa elbi ${ }^{\text {TUR }}$ & & & & & & & & & & \\
\hline Humulus lupulus L. & W & Bari sherbetit ${ }^{A L B}$ & Aerial & Infusion & Insomnia, & 3 & 3 & 1 & 0.180 & 0.563 & 0.217 & l \\
\hline $\begin{array}{l}\text { (cannabaceae) 3// } \\
\text { Pz/2013 }\end{array}$ & & Amel brumit $^{\text {TUR }}$ & & & Appetizing, & 2 & 5 & 2 & & & & \\
\hline & & $\mathrm{Amel}^{\mathrm{TUR}}$ & & & Neurorelaxant & 0 & 2 & 2 & & & & \\
\hline & & & Fruits & Infusion & Insomnia, & 6 & 5 & 3 & & & & \\
\hline & & & & & Diuretic, & 0 & 3 & 1 & & & & \\
\hline & & & & & Prostate disorders & 0 & 0 & 1 & & & & \\
\hline Hypericum perforatum & W & Kantarioni ${ }^{\mathrm{ALB}}$ & Aerial & Infusion & Anti-anemic, Wound & 0 & 4 & 0 & 0.475 & 1.844 & 0 & I \\
\hline $\begin{array}{l}\text { L. (Hypericaceae) 36/ } \\
\text { Pz/2013 08/Pz/11 }\end{array}$ & & Kantarion $^{\mathrm{BOG}}$ & parts & & $\begin{array}{l}\text { healing, } \\
\text { Anticoagulant, }\end{array}$ & 12 & 32 & 0 & & & & \\
\hline & & & & & Neurorelaxant, & 0 & 1 & 0 & & & & \\
\hline & & & & & & 16 & 21 & 0 & & & & \\
\hline & & & & & & 1 & 1 & 0 & & & & \\
\hline Inula sp. (Asteraceae) & W & Omani ${ }^{T U R}$ & Roots & Infusion & Anti-tussive, Bile & 0 & 0 & 2 & 0 & 0 & 0.130 & I \\
\hline & & Safra oti ${ }^{\text {TUR }}$ & & & simulation, Diuretic & 0 & 0 & 3 & & & & \\
\hline & & & & & & 0 & 0 & 1 & & & & \\
\hline Juglans regia L. & $\mathrm{C}$ & Arra $^{\text {ALB }}$ & Fruits & Eaten & Anti-parasitic, & 3 & 0 & 0 & 0.115 & 0 & 0 & 1 \\
\hline $\begin{array}{l}\text { (Juglandaceae) 40/ } \\
\text { Pz/2013 }\end{array}$ & & & & & Thyroid disorders & 4 & 0 & 0 & & & & \\
\hline $\begin{array}{l}\text { Juncus effusus L. } \\
\text { (Juncaceae) }\end{array}$ & W & XukllaTUR & $\begin{array}{l}\text { Aerial } \\
\text { parts }\end{array}$ & Infusion & $\begin{array}{l}\text { Urinary tract } \\
\text { disorders }\end{array}$ & 0 & 0 & 3 & 0 & 0 & 0.065 & 1 \\
\hline Juniperus communis L. & W & GëllijaALB & Wood & Extracted with & Anti-fungal, Skin & 6 & 17 & 5 & 0.623 & 2.094 & 0.696 & I \\
\hline $\begin{array}{l}\text { (cupressaceae) 39/ } \\
\text { Pz/2013 }\end{array}$ & & $\mathrm{Kleka}^{\mathrm{BOG}}$ & & $\begin{array}{l}\text { oll, topically } \\
\text { used in skin }\end{array}$ & & 2 & 11 & 2 & & & & \\
\hline & & Ardeq $^{\text {TUR }}$ & & & & & & & & & & \\
\hline & & & Fruits & $\begin{array}{l}\text { Extracted with } \\
\text { alcohols }\end{array}$ & Anti-rheumatic & 5 & 3 & 0 & & & & \\
\hline & & & & Infusion & Tuberculosis, & 6 & 2 & 9 & & & & \\
\hline & & & & & Anti-rheumatic, & 3 & 11 & 5 & & & & \\
\hline & & & & & Lithontriptic, & 9 & 21 & 11 & & & & \\
\hline & & & & & Anti-asthmatic, & 4 & 1 & 0 & & & & \\
\hline & & & & & Anti-diabetic & 3 & 1 & 0 & & & & \\
\hline Lactuca sativa $L$. & C & SallataALB & Aerial & Eaten fresh & Headache, & 0 & 0 & 2 & 0 & 0 & 0.043 & 1 \\
\hline $\begin{array}{l}\text { (Asteraceae) 41/ } \\
\text { Pz/2013 }\end{array}$ & & Marrolli ${ }^{\text {TUR }}$ & parts & & Galactogogue & 0 & 0 & 4 & & & & \\
\hline $\begin{array}{l}\text { Leonurus cardiaca L. } \\
\text { (Lamiaceae) }\end{array}$ & W & Ayslan kuyrgu ${ }^{T U R}$ & $\begin{array}{l}\text { Aerial } \\
\text { parts }\end{array}$ & Infusion & Cardiotonic, & 0 & 0 & 3 & 0 & 0 & 0.065 & I \\
\hline
\end{tabular}


Table 1 Medicinal plant used in the study area (Continued)

\begin{tabular}{|c|c|c|c|c|c|c|c|c|c|c|c|c|}
\hline & & & & & $\begin{array}{l}\text { Improve blood } \\
\text { circulation, }\end{array}$ & 0 & 0 & 2 & & & & \\
\hline & & & & & $\begin{array}{l}\text { Memory } \\
\text { enhancement }\end{array}$ & 0 & 0 & 4 & & & & \\
\hline \multirow{2}{*}{$\begin{array}{l}\text { Lycoperdon sp. } \\
\text { (Agaricaceae) 60/ } \\
\text { Pz/2013 }\end{array}$} & \multirow[t]{2}{*}{ W } & Pufka ${ }^{A L B}$ & \multirow[t]{2}{*}{ Powder } & \multirow{2}{*}{$\begin{array}{l}\text { Topically } \\
\text { applied }\end{array}$} & \multirow{2}{*}{$\begin{array}{l}\text { Wound healing, } \\
\text { Hemostatic }\end{array}$} & 4 & 1 & 0 & 0.066 & 0.031 & 0 & 1 \\
\hline & & Mantari $^{\mathrm{BOG}}$ & & & & 8 & 4 & 0 & & & & \\
\hline \multirow[t]{2}{*}{$\begin{array}{l}\text { Lycopodium clavatum } \\
\text { L. (Lycopodiaceae) }\end{array}$} & \multirow[t]{2}{*}{ W } & Bari qibritit ${ }^{A L B}$ & \multirow[t]{2}{*}{$\begin{array}{l}\text { Aerial } \\
\text { parts }\end{array}$} & $\begin{array}{l}\text { Topically } \\
\text { applied to skin }\end{array}$ & Anti-microbial & 2 & 0 & 0 & \multirow[t]{2}{*}{0.033} & \multirow[t]{2}{*}{0} & \multirow[t]{2}{*}{0} & I \\
\hline & & & & Infusion & Hepatitis & 1 & 0 & 0 & & & & \\
\hline \multirow{6}{*}{$\begin{array}{l}\text { Malva sylvestris L. } \\
\text { (Malvaceae) 44/ } \\
\text { Pz/2013 }\end{array}$} & \multirow[t]{6}{*}{ W } & Mullaga ${ }^{A L B}$ & \multirow{3}{*}{$\begin{array}{l}\text { Aerial } \\
\text { parts }\end{array}$} & \multirow{3}{*}{$\begin{array}{l}\text { Extracted with } \\
\text { fat (melhem) }\end{array}$} & Wound healing, & 3 & 5 & 2 & \multirow[t]{6}{*}{0.262} & \multirow[t]{6}{*}{0.250} & \multirow[t]{6}{*}{0.283} & I \\
\hline & & Mali slez ${ }^{\text {BOG }}$ & & & Anti-acne & 5 & 2 & 0 & & & & \\
\hline & & Ebe gumeci ${ }^{\text {TUR }}$ & & & & & & & & & & \\
\hline & & & \multirow[t]{3}{*}{ Flowers } & \multirow[t]{3}{*}{ Infusion } & Anti-tussive, & 2 & 1 & 3 & & & & \\
\hline & & & & & Bronchitis, & 2 & 0 & 4 & & & & \\
\hline & & & & & Antimicrobial & 4 & 0 & 4 & & & & \\
\hline \multirow{3}{*}{$\begin{array}{l}\text { Mespilus germanica L. } \\
\text { (Rosaceae) 47/Pz/2013 }\end{array}$} & \multirow[t]{3}{*}{ C } & MushmollaALB & \multirow{3}{*}{$\begin{array}{l}\text { Aerial } \\
\text { parts }\end{array}$} & \multirow[t]{3}{*}{ Infusion } & Anti-diarrheal, & 4 & 0 & 0 & 0.115 & 0 & 0 & I \\
\hline & & & & & Anti-diabetic, & 2 & 0 & 0 & & & & \\
\hline & & & & & Ear disorders & 1 & 0 & 0 & & & & \\
\hline Melissa officinalis L. & W & Bari i bletës ${ }^{A L B}$ & Aerial & Infusion & Neurorelaxant, & 3 & 8 & 5 & 0.475 & 0.406 & 0.413 & 1 \\
\hline $\begin{array}{l}\text { (Lamiaceae) 42/ } \\
\text { Pz/2013 }\end{array}$ & & Matoqına $^{\mathrm{BOG}}$ & parts & & Headache, & 3 & 1 & 4 & & & & \\
\hline & & Molshvatrava ${ }^{\mathrm{BOG}}$ & & & Anti-hypertensive, & 2 & 0 & 1 & & & & \\
\hline & & & & & Appetizing, & 6 & 0 & 1 & & & & \\
\hline & & & & & $\begin{array}{l}\text { Improve blood } \\
\text { circulation, }\end{array}$ & & & & & & & \\
\hline & & & & & Bronchitis, & 3 & 0 & 1 & & & & \\
\hline & & & & & Anti-anemic, & 7 & 2 & 3 & & & & \\
\hline & & & & & Anti-hallucinogenic, & 0 & 1 & 3 & & & & \\
\hline & & & & & $\begin{array}{l}\text { Respiratory } \\
\text { disorders }\end{array}$ & 5 & 1 & 1 & & & & \\
\hline Mentha longifolia (L.) & $C$ & Çaj nana ${ }^{A L B}$ & Aerial & Infusion & Stomach disorders, & 0 & 3 & 0 & 0.311 & 0.75 & 0 & I \\
\hline $\begin{array}{l}\text { Pz/2013 } \\
\text { Pzacede) 45/ }\end{array}$ & & Nan & parts & & Carminative, & 3 & 4 & 0 & & & & \\
\hline & & & & & Influenza, & 2 & 6 & 0 & & & & \\
\hline & & & & & $\begin{array}{l}\text { Respiratory system } \\
\text { infections, }\end{array}$ & 8 & 9 & 0 & & & & \\
\hline & & & & & Anti-tussive, & 4 & 1 & 0 & & & & \\
\hline & & & & & Expectorant & 2 & 1 & 0 & & & & \\
\hline Mentha pulegium L. & W & Divla menta ${ }^{\mathrm{BOG}}$ & Aerial & Infusion & Neurorelaxant, & 0 & 3 & 0 & 0 & 0.625 & 0 & । \\
\hline $\begin{array}{l}\mathrm{Pz} / 2013 \\
\mathrm{P}\end{array}$ & & & & & $\begin{array}{l}\text { Improve blood } \\
\text { circulation, }\end{array}$ & 0 & 7 & 0 & & & & \\
\hline & & & & & $\begin{array}{l}\text { Respiratory system } \\
\text { infections, }\end{array}$ & 0 & 9 & 0 & & & & \\
\hline & & & & & Antitussive & 0 & 1 & 0 & & & & \\
\hline Momordica charantia & C & Kudret nare ${ }^{\text {TUR }}$ & Fruits & Mixed with oil & Wound healing, & 0 & 0 & 7 & 0 & 0 & 0.435 & I \\
\hline Pz/2013 & & Sari kadak ${ }^{T U R}$ & & -ınternal use & Anti-diabetic, & 0 & 0 & 1 & & & & \\
\hline & & & & & Anti-cancer & 0 & 0 & 4 & & & & \\
\hline & & & & & $\begin{array}{l}\text { Vulnerary for burn } \\
\text { wounds }\end{array}$ & 0 & 0 & 8 & & & & \\
\hline
\end{tabular}


Table 1 Medicinal plant used in the study area (Continued)

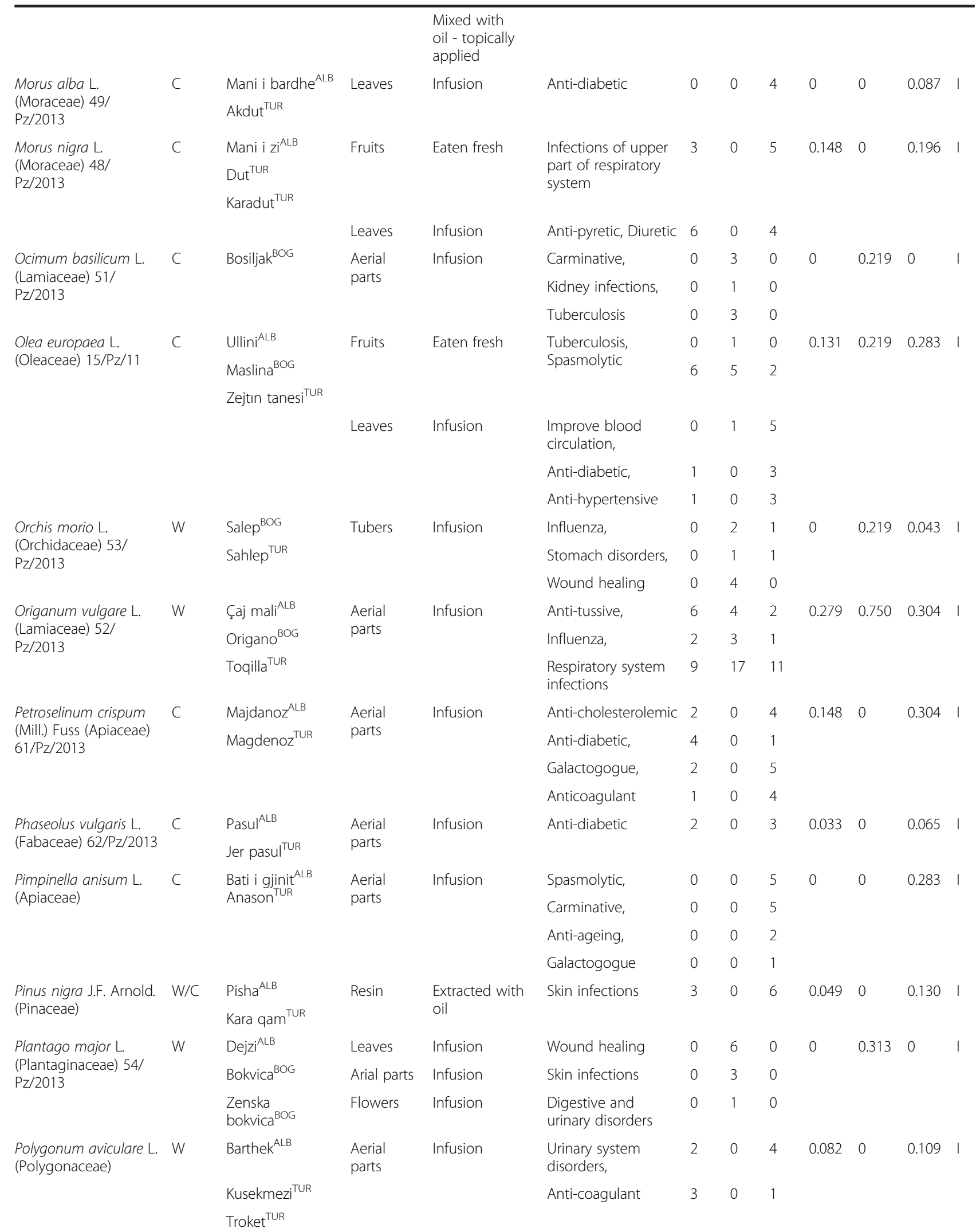


Table 1 Medicinal plant used in the study area (Continued)

\begin{tabular}{|c|c|c|c|c|c|c|c|c|c|c|c|c|}
\hline \multirow[t]{3}{*}{$\begin{array}{l}\text { Populus alba L. } \\
\text { (Salicaceae) }\end{array}$} & \multirow[t]{3}{*}{ W } & Plepi $^{\text {ALB }}$ & $\begin{array}{l}\text { Aerial } \\
\text { parts }\end{array}$ & Topically uses & Wound healing & 0 & 0 & 3 & \multirow[t]{3}{*}{0} & \multirow[t]{3}{*}{0} & \multirow[t]{3}{*}{0.087} & I \\
\hline & & Ak kavak ${ }^{T U R}$ & \multirow[t]{2}{*}{ Leaves } & Infusion & \multirow{2}{*}{$\begin{array}{l}\text { Urinary tract } \\
\text { disorders }\end{array}$} & \multirow[t]{2}{*}{0} & \multirow[t]{2}{*}{0} & \multirow[t]{2}{*}{1} & & & & \\
\hline & & Beyaz kavak'TUR & & & & & & & & & & \\
\hline \multirow{8}{*}{$\begin{array}{l}\text { Primula veris } \mathrm{L} \text {. } \\
\text { (Primulaceae) 56/ } \\
\text { Pz/2013 }\end{array}$} & \multirow[t]{8}{*}{ W } & $\begin{array}{l}\text { Myzhdja e } \\
\text { pranverës }^{A L B}\end{array}$ & \multirow[t]{5}{*}{ Flowers } & \multirow[t]{5}{*}{ Infusion } & Headache, & 0 & 2 & 1 & \multirow[t]{8}{*}{0.508} & \multirow[t]{8}{*}{1.094} & \multirow[t]{8}{*}{0.543} & I \\
\hline & & Jaglika ${ }^{\mathrm{BOG}}$ & & & Anti-tussive, & 11 & 14 & 9 & & & & \\
\hline & & Zuti vet ${ }^{\mathrm{BOG}}$ & & & $\begin{array}{l}\text { Respiratory system } \\
\text { disorders, }\end{array}$ & 14 & 7 & 9 & & & & \\
\hline & & Jagorcevina ${ }^{\mathrm{BOG}}$ & & & Improve blood & 3 & 1 & 1 & & & & \\
\hline & & Quha çicegi ${ }^{\text {TUR }}$ & & & circulation & & & & & & & \\
\hline & & & \multirow{3}{*}{$\begin{array}{l}\text { Aerial } \\
\text { parts }\end{array}$} & \multirow[t]{3}{*}{ Infusion } & Anti-tussive, & 2 & 4 & 0 & & & & \\
\hline & & & & & Expectorant, & 1 & 6 & 4 & & & & \\
\hline & & & & & Bronchitis & 0 & 1 & 1 & & & & \\
\hline \multirow{5}{*}{$\begin{array}{l}\text { Prunus avium L. } \\
\text { (Rosaceae) }\end{array}$} & \multirow[t]{5}{*}{ C } & QershiaALB & \multirow[t]{4}{*}{ Fruits } & \multirow[t]{4}{*}{ Decoction } & Anti-hypertensive, & 2 & 0 & 0 & 0.262 & 0 & 0 & 1 \\
\hline & & & & & $\begin{array}{l}\text { Improve blood } \\
\text { circulation, }\end{array}$ & 5 & 0 & 0 & & & & \\
\hline & & & & & Anti-bacterial, & 3 & 0 & 0 & & & & \\
\hline & & & & & $\begin{array}{l}\text { Digestive tract } \\
\text { disorders }\end{array}$ & 1 & 0 & 0 & & & & \\
\hline & & & Resin & Topically used & Scabies & 5 & 0 & 0 & & & & \\
\hline Prunus domestica L. & C & Sljiva ${ }^{B O G}$ & Fruits & Decoction & Hepatic disorders, & 0 & 1 & 0 & 0 & 0.438 & 0 & I \\
\hline (Rosaceae) 55/Pz/2013 & & & & & Anti-hemorrhoidal, & 0 & 5 & 0 & & & & \\
\hline & & & & & Anti-parasitic, & 0 & 2 & 0 & & & & \\
\hline & & & & & Constipation & 0 & 6 & 0 & & & & \\
\hline Prunus spinosa L. & W & Kulumrija ${ }^{A L B}$ & Flowers & Infusion & Constipation & 0 & 3 & 1 & 0.131 & 0 & 0.065 & I \\
\hline & & Ternina $^{\mathrm{BOG}}$ & Fruits & Infusion & Anti-diabetic, & 7 & 2 & 4 & & & & \\
\hline & & Kurumlia ${ }^{\text {TUR }}$ & & & Hepatic disorders & 0 & 1 & 1 & & & & \\
\hline & & & Leaves & Infusion & Improve digestion, & 1 & 1 & 2 & & & & \\
\hline & & & & & Appetizing & 0 & 2 & 1 & & & & \\
\hline Pteridium aquilinum & W & Firi $^{A L B}$ & Leaves & Extracted with & Wound healing & 0 & 0 & 3 & 0 & 0 & 0.065 & I \\
\hline $\begin{array}{l}\text { (L.) Kuhn. } \\
\text { (Dennstaedtiaceae) }\end{array}$ & & Qiban otu ${ }^{\text {TUR }}$ & & & & & & & & & & \\
\hline Pulmonaria officinalis & W & Bar I & Aerial & Infusion & Anti-tussive, & 1 & 3 & 0 & 0.016 & 0.125 & 0 & I \\
\hline & & Pluqnjak ${ }^{B O G}$ & & & Bronchitis & 0 & 1 & 0 & & & & \\
\hline Pyrus communis L. & W & Dardha ${ }^{\text {ALB }}$ & Fruits & Infusion & Cardiotonic, & 0 & 3 & 0 & 0 & 0.125 & 0.043 & । \\
\hline (Rosaceae) 58/Pz/2013 & & Dardha eger ${ }^{A L B}$ & & & Hepatic disorders & 0 & 1 & 2 & & & & \\
\hline & & Armut $^{\text {TUR }}$ & & & & & & & & & & \\
\hline $\begin{array}{l}\text { Raphanus sativus L. } \\
\text { (Brassicaceae) 63/ }\end{array}$ & C & Rotkva ${ }^{\mathrm{BOG}}$ & Taproot & Infusion & $\begin{array}{l}\text { Digestive system } \\
\text { infections, }\end{array}$ & 0 & 2 & 0 & 0 & 0.375 & 0 & I \\
\hline Pz/2013 & & Cvekla ${ }^{\mathrm{BOG}}$ & & & Bronchitis, & 0 & 4 & 0 & & & & \\
\hline & & & & & Anti-anemic, & 0 & 1 & 0 & & & & \\
\hline & & & & & Anti-rheumatic & 0 & 5 & 0 & & & & \\
\hline Ribes rubrum L. & C & Ribizla ${ }^{\mathrm{BOG}}$ & Fruits & Infusion & Anti-rheumatic, & 0 & 3 & 0 & 0 & 0.344 & 0 & I \\
\hline (Grossulanacede) & & & & & Anti-malarial, & 0 & 1 & 0 & & & & \\
\hline & & & & & Anti-allergic, & 0 & 2 & 0 & & & & \\
\hline & & & & & Heart disorders & 0 & 5 & 0 & & & & \\
\hline
\end{tabular}


Table 1 Medicinal plant used in the study area (Continued)

\begin{tabular}{|c|c|c|c|c|c|c|c|c|c|c|c|c|}
\hline \multirow{2}{*}{$\begin{array}{l}\text { Robinia pseudoacacia } \\
\text { L. (Fabaceae) 68/ } \\
\text { Pz/2013 }\end{array}$} & \multirow[t]{2}{*}{ W } & Bagremi $^{\text {ALB }}$ & \multirow[t]{2}{*}{ Flowers } & \multirow[t]{2}{*}{ Infusion } & \multirow[t]{2}{*}{ Skin infections } & \multirow[t]{2}{*}{3} & \multirow[t]{2}{*}{0} & \multirow[t]{2}{*}{2} & \multirow[t]{2}{*}{0.049} & \multirow[t]{2}{*}{0} & \multirow[t]{2}{*}{0.043} & \multirow[t]{2}{*}{ I } \\
\hline & & Akasya $^{\text {TUR }}$ & & & & & & & & & & \\
\hline \multirow{5}{*}{$\begin{array}{l}\text { Rosa canina L. } \\
\text { (Rosaceae) 67/Pz/2013 }\end{array}$} & \multirow[t]{5}{*}{ W } & Kaça ${ }^{A L B}$ & \multirow[t]{5}{*}{ Fruits } & \multirow[t]{5}{*}{ Infusion } & Improve immunity, & 3 & 1 & 0 & \multirow[t]{5}{*}{0.328} & \multirow[t]{5}{*}{0.594} & \multirow[t]{5}{*}{0} & \multirow[t]{5}{*}{ I } \\
\hline & & Shipak $^{\mathrm{BOG}}$ & & & Hepatic disorders, & 1 & 2 & 0 & & & & \\
\hline & & Sipurak ${ }^{\mathrm{BOG}}$ & & & Anti-anaemic, & 1 & 5 & 0 & & & & \\
\hline & & & & & Influenza, & 6 & 3 & 0 & & & & \\
\hline & & & & & $\begin{array}{l}\text { Digestive tract } \\
\text { disorders. }\end{array}$ & 9 & 8 & 0 & & & & \\
\hline \multirow{3}{*}{$\begin{array}{l}\text { Rubia tinctorum L. } \\
\text { (Rubiaceae) }\end{array}$} & \multirow[t]{3}{*}{ W } & Crvenka $^{\text {BOG }}$ & \multirow{3}{*}{$\begin{array}{l}\text { Aerial } \\
\text { parts }\end{array}$} & \multirow[t]{3}{*}{ Infusion } & Kidney disorders, & 0 & 3 & 0 & 0 & 0.281 & 0 & I \\
\hline & & & & & $\begin{array}{l}\text { Skeletal disorders, } \\
\text { tuberculosis }\end{array}$ & 0 & 1 & 0 & & & & \\
\hline & & & & & $\begin{array}{l}\text { "Saraxha" } \\
\text { (cutaneous } \\
\text { tuberculosis) }\end{array}$ & 0 & 5 & 0 & & & & \\
\hline Rubus fruticosus $\mathrm{L}$. & W & Mana ${ }^{A L B}$ & Aerial & Infusion & Anti-anemic, & 0 & 3 & 0 & 0 & 1.031 & 0 & । \\
\hline & & $K_{\text {Kupina }}{ }^{B O G}$ & parts & & $\begin{array}{l}\text { Improve blood } \\
\text { circulation, }\end{array}$ & 0 & 1 & 0 & & & & \\
\hline & & & & & Anti-hypertensive, & 0 & 4 & 0 & & & & \\
\hline & & & & & Wound healing & 0 & 3 & 0 & & & & \\
\hline & & & & & Anti-diabetic, & 0 & 4 & 0 & & & & \\
\hline & & & & & Antimycotic & 0 & 1 & 0 & & & & \\
\hline & & & Fruits & Infusion & Anti-anemic, & 0 & 4 & 0 & & & & \\
\hline & & & & & Anti- diarrheal, & 0 & 1 & 0 & & & & \\
\hline & & & & & Kidney infections, & 0 & 2 & 0 & & & & \\
\hline & & & & & $\begin{array}{l}\text { Oral cavity } \\
\text { infections, }\end{array}$ & 0 & 5 & 0 & & & & \\
\hline & & & & & Hypertensive, & 0 & 2 & 0 & & & & \\
\hline & & & & & Anti-parasitic, & 0 & 1 & 0 & & & & \\
\hline & & & & & Anti-tussive & 0 & 2 & 0 & & & & \\
\hline $\begin{array}{l}\text { Rubus idaeus L. } \\
\text { (Rosaceae) 66/Pz/2013 }\end{array}$ & W & Mjedra ${ }^{A L B}$ & Leaves & Infusion & $\begin{array}{l}\text { Improve blood } \\
\text { circulation, }\end{array}$ & 0 & 5 & 0 & 0 & 1.188 & 0 & 1 \\
\hline & & Malina ${ }^{\mathrm{BOG}}$ & & & Anti-hypertensive, & 0 & 5 & 0 & & & & \\
\hline & & & & & Anti-diarrheal, & 0 & 3 & 0 & & & & \\
\hline & & & & & Anti-tussive, & 0 & 2 & 0 & & & & \\
\hline & & & & & Anti-pyretic, & 0 & 1 & 0 & & & & \\
\hline & & & & & $\begin{array}{l}\text { Oral cavity } \\
\text { infections }\end{array}$ & 0 & 5 & 0 & & & & \\
\hline & & & Roots & Infusion & Anti-hypertensive, & 0 & 3 & 0 & & & & \\
\hline & & & & & Wound healing & 0 & 4 & 0 & & & & \\
\hline & & & Fruits & Infusion & Dysentery, & 0 & 1 & 0 & & & & \\
\hline & & & & & Tonsillitis, & 0 & 3 & 0 & & & & \\
\hline & & & & & Digestive disorders & 0 & 2 & 0 & & & & \\
\hline & & & Flowers & $\begin{array}{l}\text { Extracted with } \\
\text { olive oil }\end{array}$ & $\begin{array}{l}\text { To treated skin } \\
\text { wounds caused by } \\
\text { insects and snakes }\end{array}$ & 0 & 4 & 0 & & & & \\
\hline Salix alba L. & W & $\mathrm{Vrba}^{\mathrm{BOG}}$ & Leaves & Infusion & Hepatic disorders & 0 & 3 & 0 & 0 & 0.313 & 0 & I \\
\hline $\begin{array}{l}\text { (salıcaceae) /0/ } \\
\text { Pz/2013 }\end{array}$ & & & Cortex & Infusion & Antipyretic, & 0 & 5 & 0 & & & & \\
\hline
\end{tabular}


Table 1 Medicinal plant used in the study area (Continued)

\begin{tabular}{|c|c|c|c|c|c|c|c|c|c|c|c|c|}
\hline & & & & & Analgesic & 0 & 2 & 0 & & & & \\
\hline \multirow[t]{3}{*}{$\begin{array}{l}\text { Salvia officinalis L. } \\
\text { (Lamiaceae) }\end{array}$} & \multirow[t]{3}{*}{ C } & \multirow[t]{3}{*}{ Zalfija $^{\text {BOG }}$} & \multirow[t]{3}{*}{$\begin{array}{l}\text { Aerial } \\
\text { parts }\end{array}$} & \multirow[t]{3}{*}{$\begin{array}{l}\text { Infusion, then } \\
\text { added honey }\end{array}$} & $\begin{array}{l}\text { Tonsillitis and other } \\
\text { infection of } \\
\text { respiratory system, }\end{array}$ & 0 & 7 & 0 & \multirow[t]{3}{*}{0} & \multirow[t]{3}{*}{0.344} & \multirow[t]{3}{*}{0} & \multirow[t]{3}{*}{ । } \\
\hline & & & & & Anti-diabetic & 0 & 2 & 0 & & & & \\
\hline & & & & & Antiperspirant & 0 & 2 & 0 & & & & \\
\hline \multirow{11}{*}{$\begin{array}{l}\text { Sambucus nigra L. } \\
\text { (Adoxaceae) 69/ } \\
\text { Pz/2013 }\end{array}$} & \multirow[t]{11}{*}{ W } & Shtogu ${ }^{A L B}$ & \multirow[t]{11}{*}{ Flowers } & \multirow[t]{9}{*}{ Infusion } & Bronchitis, & 14 & 7 & 15 & \multirow[t]{11}{*}{0.787} & \multirow[t]{11}{*}{1.250} & \multirow[t]{11}{*}{0.891} & \multirow[t]{11}{*}{1} \\
\hline & & Zova $^{\text {BOG }}$ & & & Anti-tussive, & 8 & 5 & 3 & & & & \\
\hline & & Bos zova ${ }^{\text {BOG }}$ & & & $\begin{array}{l}\text { Expectorant, } \\
\text { Antiperspirant, }\end{array}$ & 3 & 7 & 8 & & & & \\
\hline & & Murver $^{T U R}$ & & & Anti-halitosis, & 2 & 1 & 4 & & & & \\
\hline & & Forboz $^{\text {TUR }}$ & & & Influenza, & 0 & 1 & 4 & & & & \\
\hline & & & & & Anti-asthmatic, & 6 & 8 & 2 & & & & \\
\hline & & & & & Stomach disorders, & 9 & 4 & 1 & & & & \\
\hline & & & & & $\begin{array}{l}\text { Urinary tract } \\
\text { disorders }\end{array}$ & 4 & 1 & 1 & & & & \\
\hline & & & & & & 1 & 1 & 1 & & & & \\
\hline & & & & $\begin{array}{l}\text { Extracted with } \\
\text { fish oil }\end{array}$ & $\begin{array}{l}\text { Anti-anemic, } \\
\text { Improve immunity }\end{array}$ & 0 & 3 & 1 & & & & \\
\hline & & & & $\begin{array}{l}\text { Extracted with } \\
\text { oil - topically } \\
\text { used }\end{array}$ & $\begin{array}{l}\text { Vulnerary for burns, } \\
\text { skin infections }\end{array}$ & 1 & 2 & 1 & & & & \\
\hline \multirow{6}{*}{$\begin{array}{l}\text { Satureja montana L. } \\
\text { (Lamiaceae) 19/Pz/11 }\end{array}$} & \multirow[t]{6}{*}{ W } & Cubar $^{\text {TUR }}$ & \multirow{6}{*}{$\begin{array}{l}\text { Aerial } \\
\text { parts }\end{array}$} & \multirow[t]{6}{*}{ Infusion } & Spasmolytic, & 0 & 5 & 0 & \multirow[t]{6}{*}{0} & \multirow[t]{6}{*}{0.563} & \multirow[t]{6}{*}{0} & \multirow[t]{6}{*}{ । } \\
\hline & & Curbice $^{\mathrm{BOG}}$ & & & Anti-diabetic, & 0 & 2 & 0 & & & & \\
\hline & & & & & Anti-parasitic, & 0 & 2 & 0 & & & & \\
\hline & & & & & $\begin{array}{l}\text { Respiratory tract } \\
\text { infections, }\end{array}$ & 0 & 5 & 0 & & & & \\
\hline & & & & & Anti-tussive, & 0 & 2 & 0 & & & & \\
\hline & & & & & Expectorant & 0 & 2 & 0 & & & & \\
\hline $\begin{array}{l}\text { Scrophularia nodosa L. } \\
\text { (Scrophulariaceae) }\end{array}$ & W & / & $\begin{array}{l}\text { Aerial } \\
\text { parts }\end{array}$ & $\begin{array}{l}\text { Topically } \\
\text { applied }\end{array}$ & $\begin{array}{l}\text { "Saraxha" } \\
\text { (cutaneous } \\
\text { tuberculosis), } \\
\text { Tuberculosis }\end{array}$ & 0 & 3 & 2 & 0 & 0.094 & 0.043 & I \\
\hline \multirow[t]{2}{*}{$\begin{array}{l}\text { Sempervivum tectorum } \\
\text { L. (Crassulaceae) } 71 / \\
\text { Pz/2013 }\end{array}$} & \multirow[t]{2}{*}{ W } & $\begin{array}{l}\text { Bar veshi }{ }^{\mathrm{ALB}} \\
\text { Cuvarkuca }^{\mathrm{BOG}}\end{array}$ & \multirow[t]{2}{*}{ Leaves } & $\begin{array}{l}\text { Extracted with } \\
\text { fat (cow or pig } \\
\text { fat) - topically } \\
\text { applied }\end{array}$ & Wound healing & 6 & 3 & 0 & 0.279 & 0.531 & 0 & । \\
\hline & & & & $\begin{array}{l}\text { Juice from fresh } \\
\text { leaves, } 2-3 \\
\text { drops }\end{array}$ & $\begin{array}{l}\text { Earache, ear } \\
\text { infections }\end{array}$ & 11 & 14 & 0 & & & & \\
\hline $\begin{array}{l}\text { Symphytum officinale } \\
\text { L. (Boraginaceae) 73/ }\end{array}$ & W & $\begin{array}{l}\text { Crni gavez } \\
\text { Ganez }\end{array}$ & Roots & $\begin{array}{l}\text { Extracted with } \\
\text { fat }\end{array}$ & Wound healing & 0 & 4 & 2 & 0 & 0.188 & 0.109 & । \\
\hline PZ/2013 & & & & $\begin{array}{l}\text { Extracted with } \\
\text { wine }\end{array}$ & Anticoagulant & 0 & 2 & 3 & & & & \\
\hline $\begin{array}{l}\text { Tanacetum vulgare L. } \\
\text { (Asteraceae) 75/ }\end{array}$ & W & Pire otu ${ }^{T U R R}$ & Seeds & Infusion & $\begin{array}{l}\text { Anti-parasitic } \\
\text { (intestinal parasites), }\end{array}$ & 0 & 0 & 3 & 0 & 0 & 0.304 & । \\
\hline 2013 & & & & & Anti-rheumatic & 0 & 0 & 2 & & & & \\
\hline & & & Flowers & Powder & $\begin{array}{l}\text { Insect repellent, } \\
\text { anti-parasitic }\end{array}$ & 0 & 0 & 4 & & & & \\
\hline & & & $\begin{array}{l}\text { Aerial } \\
\text { parts }\end{array}$ & Infusion & $\begin{array}{l}\text { Digestive tract } \\
\text { disorders, }\end{array}$ & 0 & 0 & 2 & & & & \\
\hline
\end{tabular}


Table 1 Medicinal plant used in the study area (Continued)

\begin{tabular}{|c|c|c|c|c|c|c|c|c|c|c|c|c|}
\hline & & & & & Anti-hemorrhoidal, & 0 & 0 & 1 & & & & \\
\hline & & & & & Eczema & 0 & 0 & 2 & & & & \\
\hline \multirow{6}{*}{$\begin{array}{l}\text { Taraxacum officinale } \\
\text { F.H. Wigg. } \\
\text { (Asteraceae)84/ } \\
\text { Pz/2013 }\end{array}$} & \multirow[t]{6}{*}{ W } & & Flowers & Infusion & Hepatitis & 2 & 1 & 0 & 0.295 & 0.375 & 0.304 & 1 \\
\hline & & & \multirow{5}{*}{$\begin{array}{l}\text { Aerial } \\
\text { parts }\end{array}$} & \multirow[t]{5}{*}{ Infusion } & Improve blood & 7 & 4 & 9 & & & & \\
\hline & & Maslacak $^{\mathrm{BOG}}$ & & & & & & & & & & \\
\hline & & Karaındıba ${ }^{T U R}$ & & & $\begin{array}{l}\text { Digestive tract } \\
\text { disorders, }\end{array}$ & 3 & 5 & 1 & & & & \\
\hline & & & & & $\begin{array}{l}\text { Urinary tract } \\
\text { disorders, }\end{array}$ & 1 & 1 & 1 & & & & \\
\hline & & & & & Anti-anemic. & 5 & 1 & 3 & & & & \\
\hline \multirow{4}{*}{$\begin{array}{l}\text { Teucrium chamaedrys } \\
\text { L. (Lamiaceae) 79/ } \\
\text { Pz/2013 }\end{array}$} & \multirow[t]{4}{*}{ W } & \multirow[t]{4}{*}{ Mamudia $^{\mathrm{BOG}}$} & \multirow{4}{*}{$\begin{array}{l}\text { Aerial } \\
\text { parts }\end{array}$} & \multirow[t]{4}{*}{ Infusion } & Appetizing, & 0 & 2 & 0 & \multirow[t]{4}{*}{0} & \multirow[t]{4}{*}{0.250} & \multirow[t]{4}{*}{0} & \multirow[t]{4}{*}{ I } \\
\hline & & & & & Stomachache, & 0 & 4 & 0 & & & & \\
\hline & & & & & Anti- diarrheal, & 0 & 1 & 0 & & & & \\
\hline & & & & & Anti-hemorrhoidal & 0 & 1 & 0 & & & & \\
\hline \multirow{6}{*}{$\begin{array}{l}\text { Teucrium polium L. } \\
\text { (Lamiaceae) 78/ } \\
\text { Pz/2013 }\end{array}$} & \multirow[t]{6}{*}{ W } & Bar saraxha ${ }^{A L B}$ & \multirow{6}{*}{$\begin{array}{l}\text { Aerial } \\
\text { parts }\end{array}$} & \multirow[t]{3}{*}{ Mixed with fat } & Tuberculosis, & 2 & 2 & 0 & \multirow[t]{6}{*}{0.131} & \multirow[t]{6}{*}{0.281} & \multirow[t]{6}{*}{0} & I \\
\hline & & Bar majasili ${ }^{\text {ALB }}$ & & & "Saraxha" & 4 & 2 & 0 & & & & \\
\hline & & Podobica ${ }^{\mathrm{BOG}}$ & & & $\begin{array}{l}\text { (cutaneous } \\
\text { tuberculosis) }\end{array}$ & & & & & & & \\
\hline & & & & \multirow[t]{3}{*}{ Infusion } & Anti-hemorrhoidal, & 1 & 1 & 0 & & & & \\
\hline & & & & & $\begin{array}{l}\text { igestive tract } \\
\text { disorders, }\end{array}$ & 1 & 3 & 0 & & & & \\
\hline & & & & & Stomachache & 0 & 1 & 0 & & & & \\
\hline \multirow{10}{*}{$\begin{array}{l}\text { Thymus serpyllum L. } \\
\text { (Lamiaceae) 76/ } \\
\text { Pz/2013 }\end{array}$} & \multirow[t]{10}{*}{ W } & $\begin{array}{l}\text { Majcina } \\
\text { dusica }^{\text {BOG }}\end{array}$ & $\begin{array}{l}\text { Aerial } \\
\text { parts }\end{array}$ & Infusion & $\begin{array}{l}\text { Improve blood } \\
\text { circulation, }\end{array}$ & 3 & 5 & 2 & 1.525 & 2.5 & 1.087 & I \\
\hline & & Qeklik oti $^{\text {TUR }}$ & & & Anticholesterolemic, & 1 & 2 & 0 & & & & \\
\hline & & & & & $\begin{array}{l}\text { Respiratory } \\
\text { inflammations, }\end{array}$ & 21 & 26 & 16 & & & & \\
\hline & & & & & Immunostimulant, & 4 & 0 & 2 & & & & \\
\hline & & & & & Neurorelaxant, & 11 & 5 & 3 & & & & \\
\hline & & & & & Carminative, & 19 & 22 & 13 & & & & \\
\hline & & & & & Spasmolytic, & 13 & 9 & 12 & & & & \\
\hline & & & & & Bronchitis, & 16 & 6 & 1 & & & & \\
\hline & & & & & Anti-asthmatic, & 2 & 4 & 1 & & & & \\
\hline & & & & & Expectorant & 3 & 1 & 0 & & & & \\
\hline Thymus vulgaris $L$. & W & Majcina & Aerial & Infusion & Anti-tussive, & 0 & 3 & 0 & 0 & 0.281 & 0 & I \\
\hline Pz/2013 & & dusic & parts & & Anti-cholesterolemic & 0 & 6 & 0 & & & & \\
\hline Typha latifolia L. & W & Shavar ${ }^{\text {ALB }}$ & Fruits & Infusion & Respiratory system & 0 & 0 & 3 & 0 & 0 & 0.065 & 1 \\
\hline $\begin{array}{l}\text { (Iyphaceae) 82/ } \\
\text { Pz/2013 }\end{array}$ & & Hubabo TUR & & & & & & & & & & \\
\hline Tilia platyphyllos Scop. & W/C & Blini ${ }^{A L B}$ & Flowers & Infusion & Respiratory system & 8 & 13 & 8 & 0.689 & 1.469 & 0.804 & I \\
\hline $\begin{array}{l}\text { (IMalvaceae) 80/ } \\
\text { Pz/2013 }\end{array}$ & & $\operatorname{Lipa}^{\mathrm{BOG}}$ & & & ns, & & & & & & & \\
\hline & & Flamur $^{\text {TUR }}$ & & & Anti-anemic, & 2 & 5 & 4 & & & & \\
\hline & & Ilhamur $^{\text {TUR }}$ & & & Stomach infections, & 9 & 3 & 5 & & & & \\
\hline & & & & & Headache, & 1 & 0 & 1 & & & & \\
\hline & & & & & Anti-tussive & 2 & 0 & 1 & & & & \\
\hline & & & & Infusion & Anti-tussive, & 2 & 4 & 6 & & & & \\
\hline & & & & & Expectorant, & 1 & 3 & 1 & & & & \\
\hline
\end{tabular}


Table 1 Medicinal plant used in the study area (Continued)

\begin{tabular}{|c|c|c|c|c|c|c|c|c|c|c|c|c|}
\hline & & & $\begin{array}{l}\text { Leaves } \\
\text { and } \\
\text { Flowers }\end{array}$ & & $\begin{array}{l}\text { Respiratory system } \\
\text { inflammations }\end{array}$ & 17 & 19 & 11 & & & & \\
\hline \multirow{2}{*}{$\begin{array}{l}\text { Trifolium arvense L. } \\
\text { (Fabaceae) }\end{array}$} & \multirow[t]{2}{*}{ W/C } & TërfojaALB & \multirow[t]{2}{*}{ Aerial part } & \multirow[t]{2}{*}{ Infusion } & \multirow[t]{2}{*}{ Anti-rheumatic } & \multirow[t]{2}{*}{0} & \multirow[t]{2}{*}{6} & \multirow[t]{2}{*}{0} & \multirow[t]{2}{*}{0} & \multirow[t]{2}{*}{0.188} & \multirow[t]{2}{*}{0} & \multirow[t]{2}{*}{ I } \\
\hline & & Deklina $^{\mathrm{BOG}}$ & & & & & & & & & & \\
\hline \multirow[t]{2}{*}{$\begin{array}{l}\text { Triticum vulgare L. } \\
\text { (Poaceae) 74/Pz/2013 }\end{array}$} & \multirow[t]{2}{*}{ W } & Gruni $^{A L B}$ & \multirow[t]{2}{*}{ Flour } & $\begin{array}{l}\text { Mixed with } \\
\text { hot water - } \\
\text { topically used }\end{array}$ & $\begin{array}{l}\text { Skin inflammation } \\
\text { and ulcers }\end{array}$ & 0 & 0 & 4 & \multirow[t]{2}{*}{0} & \multirow[t]{2}{*}{0} & \multirow[t]{2}{*}{0.087} & 1 \\
\hline & & Bogday $^{\text {TUR }}$ & & $\begin{array}{l}\text { Mixed with hot } \\
\text { water - internal } \\
\text { used }\end{array}$ & Anti-diarrheal & 0 & 0 & 2 & & & & \\
\hline \multirow{2}{*}{$\begin{array}{l}\text { Tussilago farfara L. } \\
\text { (Asteraceae) 83/ } \\
\text { Pz/2013 }\end{array}$} & \multirow[t]{2}{*}{ W } & \multirow[t]{2}{*}{ Potbel ${ }^{\mathrm{BOG}}$} & \multirow{2}{*}{$\begin{array}{l}\text { Aerial } \\
\text { parts }\end{array}$} & \multirow[t]{2}{*}{ Infusion } & Expectorant, & 0 & 7 & 0 & \multirow[t]{2}{*}{0} & \multirow[t]{2}{*}{0.313} & 0 & I \\
\hline & & & & & Anti-tussive & 0 & 3 & 0 & & & & \\
\hline Ulmus minor Mill. & W & $\operatorname{Vidhi}^{\mathrm{ALB}}$ & Leaves & Extracted & Anti-mycotic, & 11 & 0 & 7 & 0.197 & 0 & 0.217 & I \\
\hline & & Karragaq $^{\text {TUR }}$ & & & $\begin{array}{l}\text { Anti-bacterial, } \\
\text { "Saraxha" } \\
\text { (cutaneous } \\
\text { tuberculosis). }\end{array}$ & 1 & 0 & 3 & & & & \\
\hline Urtica dioica L. & W & Hithi $^{\text {ALB }}$ & Aerial & Infusion & Anti-hemorrhoidal, & 3 & 1 & 5 & 1.820 & 3.094 & 1.652 & $\|$ \\
\hline $\begin{array}{l}\text { (Urticaceae) 85/ } \\
\text { Pz/2013 }\end{array}$ & & Kopriva $^{\mathrm{BOG}}$ & & & Anti-anemic, & 32 & 21 & 11 & & & & \\
\hline & & Yakici TUR & & & Influenza, & 12 & 10 & 6 & & & & \\
\hline & & & & & Anti-cancer, & 1 & 0 & 0 & & & & \\
\hline & & & & & Eczemas, & 3 & 7 & 9 & & & & \\
\hline & & & & & Bronchitis, & 11 & 19 & 14 & & & & \\
\hline & & & & & Headache, & 1 & 3 & 1 & & & & \\
\hline & & & & & Anti-rheumatic, & 9 & 4 & 6 & & & & \\
\hline & & & & & Anti-bacterial, & 12 & 19 & 13 & & & & \\
\hline & & & & & Alopecia, & 5 & 1 & 1 & & & & \\
\hline & & & & & Anti-dandruff, & 18 & 12 & 9 & & & & \\
\hline & & & & & Digestive disorders & 2 & 1 & 1 & & & & \\
\hline & & & & & Urinary disorders & 2 & 1 & 0 & & & & \\
\hline Vaccinium myrtillus L. & W & Boronıca $^{\text {ALB }}$ & Fruits & Juice of fresh & Digestive tract & 6 & 9 & 5 & 0.984 & 1.563 & 1.152 & I \\
\hline & & Borovnica ${ }^{A L B}$ & & & mections, & & & & & & & \\
\hline & & & & & Anti-anemic, & 25 & 21 & 11 & & & & \\
\hline & & & & & Eye inflammations, & 1 & 0 & 5 & & & & \\
\hline & & & & & Hepatitis, & 0 & 0 & 3 & & & & \\
\hline & & & & & Digestive disorders, & 0 & 1 & 3 & & & & \\
\hline & & & & & Urinary disorders & 1 & 1 & 1 & & & & \\
\hline & & & Fruits and & Infusion & Lithontriptic, & 4 & 2 & 7 & & & & \\
\hline & & & leaves & & $\begin{array}{l}\text { Respiratory } \\
\text { inflammations, }\end{array}$ & 6 & 5 & 3 & & & & \\
\hline & & & & & Anti-anemic & 17 & 11 & 15 & & & & \\
\hline $\begin{array}{l}\text { Vaccinium vitis-idaea L. } \\
\text { (Ericaceae) }\end{array}$ & W & Brusnica $^{\mathrm{BOG}}$ & Leaves & Infusion & $\begin{array}{l}\text { Urinary } \\
\text { inflammations }\end{array}$ & 0 & 14 & 0 & 0 & 2.094 & 0 & I \\
\hline & & & & & Anti-rheumatic & 0 & 3 & 0 & & & & \\
\hline & & & Fruits & Infusion & $\begin{array}{l}\text { Urinary tract } \\
\text { infections }\end{array}$ & 0 & 21 & 0 & & & & \\
\hline & & & & Infusion & Lithontriptic, & 0 & 11 & 0 & & & & \\
\hline
\end{tabular}


Table 1 Medicinal plant used in the study area (Continued)

\begin{tabular}{|c|c|c|c|c|c|c|c|c|c|c|c|c|}
\hline & & & \multirow{6}{*}{$\begin{array}{l}\text { Fruits and } \\
\text { leaves }\end{array}$} & & Diuretic, & 0 & 6 & 0 & & & & \\
\hline & & & & & Anti-rheumatic, & 0 & 1 & 0 & & & & \\
\hline & & & & & Wound healing, & 0 & 3 & 0 & & & & \\
\hline & & & & & Antipyretic, & 0 & 1 & 0 & & & & \\
\hline & & & & & Anti-diabetic, & 0 & 6 & 0 & & & & \\
\hline & & & & & Anticonvulsant & 0 & 1 & 0 & & & & \\
\hline \multirow{2}{*}{$\begin{array}{l}\text { Veratrum album L. } \\
\text { (Melanthiaceae) }\end{array}$} & \multirow[t]{2}{*}{ W } & Shtara ${ }^{\mathrm{ALB}}$ & \multirow{2}{*}{$\begin{array}{l}\text { Aerial } \\
\text { parts }\end{array}$} & \multirow[t]{2}{*}{ Infusion } & \multirow[t]{2}{*}{ Anti-hypertensive. } & \multirow[t]{2}{*}{3} & \multirow[t]{2}{*}{5} & \multirow[t]{2}{*}{0} & \multirow[t]{2}{*}{0.049} & \multirow[t]{2}{*}{0.156} & \multirow[t]{2}{*}{0} & । \\
\hline & & Cemenika $^{\mathrm{BOG}}$ & & & & & & & & & & \\
\hline \multirow{3}{*}{$\begin{array}{l}\text { Verbascum sp. } \\
\text { (Scrophulariaceae) 89/ } \\
\text { Pz/2013 }\end{array}$} & \multirow[t]{3}{*}{ w } & Divizma $^{\text {BOG }}$ & \multirow{3}{*}{$\begin{array}{l}\text { Aerial } \\
\text { parts }\end{array}$} & \multirow{3}{*}{$\begin{array}{l}\text { Infusion and } \\
\text { Mixed with fat } \\
\text { "mehlem" }\end{array}$} & Anti-tussive, & 0 & 2 & 1 & \multirow[t]{3}{*}{0} & \multirow[t]{3}{*}{0.250} & \multirow[t]{3}{*}{0.087} & । \\
\hline & & Diviza & & & Bronchitis, & 0 & 5 & 2 & & & & \\
\hline & & Sigir kuyrugu TUR & & & $\begin{array}{l}\text { Digestive tract } \\
\text { disorders }\end{array}$ & 0 & 1 & 1 & & & & \\
\hline \multirow{3}{*}{$\begin{array}{l}\text { Veronica officinalis L. } \\
\text { (Plantaginaceae) 88/ } \\
\text { Pz/2013 }\end{array}$} & \multirow[t]{3}{*}{ W } & Paskalya otuTUR & \multirow[t]{3}{*}{ Leaves } & \multirow[t]{3}{*}{ Infusion } & Anticoagulant, & 0 & 0 & 3 & \multirow[t]{3}{*}{0} & \multirow[t]{3}{*}{0} & \multirow[t]{3}{*}{0.196} & । \\
\hline & & Yavshan otu ${ }^{\text {TUR }}$ & & & $\begin{array}{l}\text { Respiratory system } \\
\text { inflammations, }\end{array}$ & 00 & 0 & 2 & & & & \\
\hline & & & & & Wound healing & & 0 & 4 & & & & \\
\hline \multirow{8}{*}{$\begin{array}{l}\text { Vitis vinifera L. } \\
\text { (Vitaceae) 90/Pz/2013 }\end{array}$} & \multirow[t]{8}{*}{$C$} & Rrushı ${ }^{\mathrm{ALB}}$ & \multirow[t]{3}{*}{ Leaves } & Infusion & Increase immunity, & 4 & 1 & 0 & 0.311 & 0.438 & 0.435 & I \\
\hline & & Grozhgje $^{\mathrm{BOG}}$ & & & Hepatitis & 2 & 1 & 3 & & & & \\
\hline & & Siyah üzüm ${ }^{T U R}$ & & & & & & & & & & \\
\hline & & & Fruits & Eaten fresh & Anti-anemic, & 3 & 4 & 2 & & & & \\
\hline & & & & & Hepatic disorders, & 1 & 2 & 3 & & & & \\
\hline & & & & & $\begin{array}{l}\text { Urinary system } \\
\text { inflammations }\end{array}$ & 6 & 2 & 1 & & & & \\
\hline & & & Juice of & Internal used & Anti-anemic, & 1 & 1 & 10 & & & & \\
\hline & & & $\begin{array}{l}\text { fruits (semı } \\
\text { fermented) }\end{array}$ & & Anti-cholesterolemic & 2 & 3 & 1 & & & & \\
\hline Zea mays L. (Poaceae) & $C$ & Misri ALB & Female & Infusion & Urinary tract & 2 & 0 & 3 & 0.115 & 0 & 0.152 & 1 \\
\hline & & Kollomoq ${ }^{\text {ALB }}$ & & & 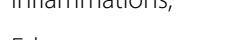 & & & & & & & \\
\hline & & & & & Edema, & 1 & 0 & 1 & & & & \\
\hline & & Kollomoqi ${ }^{\mathrm{TUR}}$ & & & Stomach disorders, & 2 & 0 & 0 & & & & \\
\hline & & & & & Anti-parasitic & 1 & 0 & 0 & & & & \\
\hline & & & Ripe seeds & Infusion & Anti-parasitic & 1 & 0 & 3 & & & & \\
\hline
\end{tabular}

${ }^{\text {a }}$ Folk Names. ${ }^{\text {ALB }}$ folk name(s) recorded among Albanians; ${ }^{B_{0}}$ folk name(s) recorded among Bosniaks/Gorani; ${ }^{\text {TUR }}$ folk name(s) recorded among Turks

${ }^{\mathbf{b}} \mathbf{A l b} \mathbf{N}_{\mathbf{u c}}$ : Number of use citations provided by Albanian informants; Bo/Go $\mathbf{N}_{\mathbf{u c}}$ : Number of use citations provided by Bosnian and Gorani informants; Tur $\mathbf{N}_{\mathbf{u c}}$ : Number of use citations provided by Turkish informants.

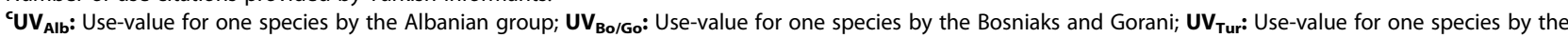
Turkish group. This index measures the relative importance of each species based on its reported use by informants from each cultural group under study.

${ }^{d} Q$ : Quadrant assignments are based on adjusted use-values ( $\left.U V_{\text {adj }}\right)$, which were calculated by dividing the use-value (UV) of each group by the maximum usevalue $\left(U V_{\max }\right)$ for medicinal citations $\left(U V_{\text {adj }}\right.$ not shown).

additional species stood out from the majority and fell into Quadrant II: Allium sativum and Urtica dioica. Both of these taxa demonstrated high use-value scores among Bosniaks/Gorani, with moderate use-values among Albanians and Turks.

Upon cross-cultural comparative analysis of our findings with those reported in the medico-ethnobotanical literature available on the Southern Balkans [1-4,6,8-10,12, $13,15,17,19-21,25-27]$, we identified the following novel uses of several plants, which could merit further phytochemical and bioactivity analyses:
- the topical application of the fruiting body of Amanita caesarea in the treatment of skin infections;

- the drinking of an infusion of the aerial parts of Apium graveolens to treat sterility;

- the drinking of an infusion of the aerial parts of Avena sativa (Figure 5)for its skeletal system enhancement effect;

- the consumption of Brassica rapa taproot to treat eye disorders and stimulate the immune system;

- the drinking of an infusion of aerial parts of Geranium sanguineum to treat respiratory disorders; 
Table 2 Wild plant or mushroom species used as local food in the study area

\begin{tabular}{|c|c|c|c|c|c|c|c|c|c|c|c|}
\hline $\begin{array}{l}\text { Botanical taxon, family } \\
\text { and voucher specimen } \\
\text { code }\end{array}$ & $\begin{array}{l}\text { Folk } \\
\text { name(s) }\end{array}$ & $\begin{array}{l}\text { Part(s) } \\
\text { used }\end{array}$ & Preparation & Folk uses(s) & $\begin{array}{l}\text { Alb } \\
\mathrm{N}_{\mathrm{uc}} \\
\mathrm{b}\end{array}$ & $\begin{array}{l}\mathrm{Bo} / \\
\mathrm{Go} \\
\mathrm{N}_{\mathrm{buc}}\end{array}$ & $\begin{array}{l}\text { Tur } \\
\mathrm{N}_{\mathrm{buc}}\end{array}$ & $\mathrm{UV}_{\mathrm{clb}}$ & $\begin{array}{l}\mathrm{UV}_{\mathrm{Bo}} \\
\mathrm{Go}_{\mathrm{C}}\end{array}$ & $U_{\text {Tur }}^{c}$ & $Q^{d}$ \\
\hline \multirow[t]{2}{*}{$\begin{array}{l}\text { Amanita caesarea (Scop.) } \\
\text { Pers. (Amanitaceae) }\end{array}$} & \multirow[t]{2}{*}{ Kërpurdha $a^{A L B}$} & \multirow[t]{2}{*}{$\begin{array}{l}\text { Aerial } \\
\text { parts }\end{array}$} & \multirow[t]{2}{*}{ Fresh or conserved } & $\begin{array}{l}\text { Food used in small } \\
\text { quantities, }\end{array}$ & 3 & 0 & 0 & 0.08 & \multirow[t]{2}{*}{0} & \multirow[t]{2}{*}{0} & I \\
\hline & & & & Food additive & 2 & 0 & 0 & 2 & & & \\
\hline $\begin{array}{l}\text { Castanea sativa Mill. } \\
\text { (Fagaceae) 19/Pz/2013 }\end{array}$ & Gështaja ${ }^{A L B}$ & Fruits & Fresh, beaked & Food & 6 & 4 & 4 & 0.098 & 0.125 & 0.087 & । \\
\hline \multirow{3}{*}{$\begin{array}{l}\text { Cichorium intybus L. } \\
\text { (Asteraceae) 21/Pz/2013 }\end{array}$} & Cikorja $^{S H Q}$ & \multirow{3}{*}{$\begin{array}{l}\text { Aerial } \\
\text { parts }\end{array}$} & \multirow[t]{3}{*}{ Dried and ground } & \multirow{3}{*}{$\begin{array}{l}\text { Coffee substitute, } \\
\text { prepared as Turkish } \\
\text { coffee }\end{array}$} & \multirow[t]{3}{*}{2} & \multirow[t]{3}{*}{0} & \multirow[t]{3}{*}{3} & \multirow[t]{3}{*}{0.033} & \multirow[t]{3}{*}{0} & \multirow[t]{3}{*}{0.065} & I \\
\hline & $\begin{array}{l}\text { Mavi } \\
\text { çiçek }\end{array}$ & & & & & & & & & & \\
\hline & $\underset{\text { TUR }}{\text { Satali bitki }}$ & & & & & & & & & & \\
\hline \multirow{3}{*}{$\begin{array}{l}\text { Cornus mas L. (Cornaceae) } \\
\text { 23/Pz/2013 }\end{array}$} & \multirow{3}{*}{$\begin{array}{l}\text { Thana } \\
\text { Dirnina }^{\text {TUR }}\end{array}$} & \multirow[t]{3}{*}{ Fruits } & Eaten fresh & Food & 5 & 6 & 0 & \multirow[t]{3}{*}{0.082} & \multirow[t]{3}{*}{0.563} & \multirow[t]{3}{*}{0} & 1 \\
\hline & & & $\begin{array}{l}\text { Mixed and boiled } \\
\text { with sugar for short } \\
\text { period }\end{array}$ & Beverage & 0 & 6 & 0 & & & & \\
\hline & & & $\begin{array}{l}\text { Mixed and boiled } \\
\text { with sugar for } \\
\text { longer period }\end{array}$ & Jam & 0 & 6 & 0 & & & & \\
\hline $\begin{array}{l}\text { Corylus avellana } \mathrm{L} \text {. } \\
\text { (Betulaceae) 24/Pz/2013 }\end{array}$ & Lejthı ALB & Fruits & Fresh or dried & Food, Sweetener & 9 & 15 & 5 & 0.148 & 0.469 & 0.109 & । \\
\hline \multirow[t]{2}{*}{$\begin{array}{l}\text { Foeniculum vulgare Mill. } \\
\text { (Apiaceae) 32/Pz/2013 }\end{array}$} & $\begin{array}{l}\text { Kopër }^{\mathrm{ALB}} \\
\text { Rezene }^{\mathrm{BOG}}\end{array}$ & \multirow[t]{2}{*}{$\begin{array}{l}\text { Leaves, } \\
\text { seeds }\end{array}$} & \multirow[t]{2}{*}{ Dried } & \multirow[t]{2}{*}{$\begin{array}{l}\text { Food additive for } \\
\text { flavoring }\end{array}$} & \multirow[t]{2}{*}{0} & \multirow[t]{2}{*}{0} & 2 & 0 & 0 & 0.043 & । \\
\hline & Anason ${ }^{\text {TUR }}$ & & & & & & & & & & \\
\hline Fragaria vesca L. (Rosaceae) & DrezaAlB & Fruits & Eaten fresh & Food & 15 & 19 & 7 & 0.295 & 1.406 & 0.196 & $\|$ \\
\hline & $\begin{array}{l}\text { Divla } \\
\text { jagoda }^{\text {BOG }}\end{array}$ & & $\begin{array}{l}\text { Mixed and boiled } \\
\text { with sugar for short } \\
\text { period }\end{array}$ & Beverage & 0 & 15 & 0 & & & & \\
\hline & & & $\begin{array}{l}\text { Mixed and boiled } \\
\text { with sugar for } \\
\text { longer period }\end{array}$ & Jam & 3 & 11 & 2 & & & & \\
\hline $\begin{array}{l}\text { Helianthus tuberosus L. } \\
\text { (Asteraceae) }\end{array}$ & Orashka ${ }^{\mathrm{ALB}}$ & Tuber & Eaten fresh & Food & 3 & 0 & 0 & 0.033 & 0.25 & 0 & । \\
\hline Malus sylvestris Mill. & & Fruits & Boiled with sugar & Jams & 0 & 3 & 0 & 0.033 & 0.25 & 0 & । \\
\hline & & & $\begin{array}{l}\text { Sliced and dried } \\
\text { (ahaf), boiled in } \\
\text { water prior to eating }\end{array}$ & Wintertime food & 2 & 5 & 0 & & & & \\
\hline Matricaria chamomilla L. & Lule qeni ${ }^{\mathrm{ALB}}$ & Aerial & Dried & Tea & 0 & 5 & 3 & 0 & 0.156 & 0.065 & । \\
\hline & Kamomil $^{\mathrm{ALB}}$ & & & & & & & & & & \\
\hline & Papatja ${ }^{T U R}$ & & & & & & & & & & \\
\hline & Sari çiçek ${ }^{T U R}$ & & & & & & & & & & \\
\hline & Kamilica $^{\mathrm{BOG}}$ & & & & & & & & & & \\
\hline & Babunec $^{\mathrm{BOG}}$ & & & & & & & & & & \\
\hline $\begin{array}{l}\text { Mentha longifolia (L.) Huds. } \\
\text { (Lamiaceae) 45/Pz/2013 }\end{array}$ & $\begin{array}{l}\text { Çaj nana }{ }^{A L B} \\
\text { Nana }\end{array}$ & $\begin{array}{l}\text { Aerial } \\
\text { parts }\end{array}$ & Infusion & Tea & 0 & 0 & 3 & 0 & 0 & 0.065 & । \\
\hline $\begin{array}{l}\text { Morus alba L. (Moraceae) 49/ } \\
\text { Pz/2013 }\end{array}$ & $\begin{array}{l}\text { Mani i } \\
\text { bardhe }\end{array}$ & Fruits & Fresh & Food & 6 & 5 & 0 & 0.098 & 0.156 & 0 & । \\
\hline & Akdut $^{\text {TUR }}$ & & & & & & & & & & \\
\hline Morus nigra L. (Moraceae) & Mani i zi ${ }^{\mathrm{ALB}}$ & Fruits & Eaten fresh & Food & 8 & 9 & 4 & 0.131 & 0.406 & 0.109 & । \\
\hline 48/Pz/201 & Dut $^{\text {TUR }}$ & & & Beverage & 0 & 2 & 1 & & & & \\
\hline
\end{tabular}


Table 2 Wild plant or mushroom species used as local food in the study area (Continued)

\begin{tabular}{|c|c|c|c|c|c|c|c|c|c|c|c|}
\hline & Karadut $^{\text {TUR }}$ & & $\begin{array}{l}\text { Mixed and boiled } \\
\text { with sugar for short } \\
\text { period }\end{array}$ & & & & & & & & \\
\hline & & & $\begin{array}{l}\text { Mixed and boiled } \\
\text { with sugar for } \\
\text { longer period }\end{array}$ & Jam & 0 & 2 & 0 & & & & \\
\hline $\begin{array}{l}\text { Orchis morio L. } \\
\text { (Orchidaceae) 53/Pz/2013 }\end{array}$ & Salepi ${ }^{A L B}$ & Tubers & Dried & $\begin{array}{l}\text { Hot beverage mixed } \\
\text { with milk "salep" }\end{array}$ & 0 & 6 & 11 & 0 & 0.188 & 0.239 & I \\
\hline Origanum vulgare L. & Çaj mali ${ }^{A L B}$ & Aerial & Infusion & Tea, Food flavoring & 8 & 13 & 0 & 0.131 & 0.406 & 0 & । \\
\hline (Lamiaceae) 52/Pz/2013 & Toqilla ${ }^{\text {TUR }}$ & parts & & & & & & & & & \\
\hline Prunus spinosa L. (Rosaceae) & Kulumrija ${ }^{A L B}$ & Fruits & Eaten fresh & Food & 4 & 8 & 0 & 0.066 & 0.25 & 0 & । \\
\hline & Ternina $^{\mathrm{BOG}}$ & & & & & & & & & & \\
\hline & Kurumlia $^{\text {TUR }}$ & & & & & & & & & & \\
\hline & $\begin{array}{l}\text { Dardha } \\
\text { egerALB }\end{array}$ & & & & & & & & & & \\
\hline Rosa canina L. (Rosaceae) & KaçaALB & Fruits & Infusion & Tea, Jam & 0 & 6 & 4 & 0 & 0.188 & 0.087 & । \\
\hline & Shipak $^{\mathrm{BOG}}$ & & & & & & & & & & \\
\hline & Sipurak $^{\mathrm{BOG}}$ & & & & & & & & & & \\
\hline $\begin{array}{l}\text { Rosa damascena Mill. } \\
\text { (Rosaceae) }\end{array}$ & Trendafili ${ }^{\mathrm{ALB}}$ & Flowers & $\begin{array}{l}\text { Flowers mixed with } \\
\text { cold water for } \\
24 \text { hours, and then } \\
\text { lemon juice is } \\
\text { added }\end{array}$ & Juice (shurup) & 5 & 0 & 0 & 0.082 & 0 & 0 & । \\
\hline Rubus fruticosus L. & ManaALB & Fruits & Eaten fresh & Food & 6 & 5 & 2 & 0.098 & 0.281 & 0.043 & । \\
\hline (Rosaceae) 65/Pz/2013 & $K_{\text {Kupina }}{ }^{\text {BOG }}$ & & $\begin{array}{l}\text { Mixed and boiled } \\
\text { with sugar for short } \\
\text { period }\end{array}$ & Beverage & 0 & 4 & 0 & & & & \\
\hline Rubus idaeus L. (Rosaceae) & MjedraALB & Fruits & Eaten fresh & Food & 5 & 4 & 4 & 0.082 & 0.219 & 0.109 & । \\
\hline 66/Pz/2013 & Malina ${ }^{\mathrm{BOG}}$ & & $\begin{array}{l}\text { Mixed and boiled } \\
\text { with sugar for short } \\
\text { period }\end{array}$ & Beverage & 0 & 3 & 1 & & & & \\
\hline Sambucus nigra $L$. & Shtogu ${ }^{\mathrm{ALB}}$ & Flowers & Flowers mixed with & Beverage & 9 & 11 & 8 & 0.148 & 0.344 & 0.174 & । \\
\hline (Adoxaceae) 69/Pz/2013 & $Z_{\text {Zva }}{ }^{\mathrm{BOG}}$ & & $\begin{array}{l}\text { cold water for } \\
24 \text { hours, and then }\end{array}$ & & & & & & & & \\
\hline & BOG zova ${ }^{\text {BOG }}$ & & lemon juice is & & & & & & & & \\
\hline & Murver $^{T U R}$ & & & & & & & & & & \\
\hline & Forboz $^{\text {TUR }}$ & & & & & & & & & & \\
\hline Thymus serpyllum L. & Qeklik oti ${ }^{\text {TUR }}$ & Aerial & Infusion & Tea & 0 & 13 & 0 & 0 & 0.406 & 0 & । \\
\hline (Lamiaceae) 76/Pz/2013 & $\begin{array}{l}\text { Majcina } \\
\text { dushica }^{\text {BOG }}\end{array}$ & & & & & & & & & & \\
\hline $\begin{array}{l}\text { Thymus vulgaris L. } \\
\text { (Lamiaceae) 77/Pz/2013 }\end{array}$ & $\begin{array}{l}\text { Majcina } \\
\text { dushica }^{\text {BOG }}\end{array}$ & $\begin{array}{l}\text { Aerial } \\
\text { parts }\end{array}$ & Infusion & Tea & 0 & 8 & 0 & 0 & 0.25 & 0 & । \\
\hline Tilia platyphyllos Scop. & Blini ${ }^{\mathrm{ALB}}$ & Flowers & Infusion & Tea & 9 & 11 & 6 & 0.148 & 0.344 & 0.13 & । \\
\hline (Malvaceae) 80/Pz/2013 & Flamur $^{T U R}$ & & & & & & & & & & \\
\hline & Ilhamur ${ }^{T U R}$ & & & & & & & & & & \\
\hline & $\operatorname{Lipa}^{\text {BOG }}$ & & & & & & & & & & \\
\hline Urtica dioica L. (Urticaceae) & Hithi $^{\text {ALB }}$ & Aerial & Young and fresh & Filling in home-made & 14 & 11 & 11 & 0.23 & 0.344 & 0.239 & । \\
\hline 86/Pz/2013 & Kopriva $^{\mathrm{BOG}}$ & parts & & savory pies (pite) & & & & & & & \\
\hline & Yakici $^{\text {TUR }}$ & & & & & & & & & & \\
\hline Vaccinium myrtillus L. & Boronıca $^{\mathrm{ALB}}$ & Fruits & Eaten fresh & Food & 8 & 11 & 5 & 0.131 & 0.594 & 0.109 & | \\
\hline
\end{tabular}


Table 2 Wild plant or mushroom species used as local food in the study area (Continued)

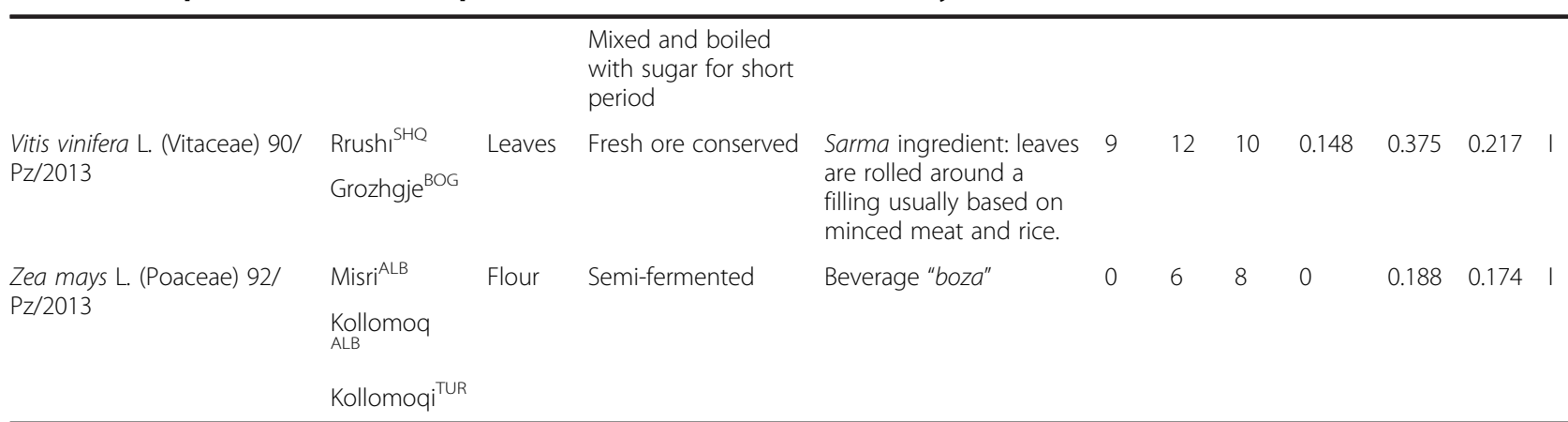

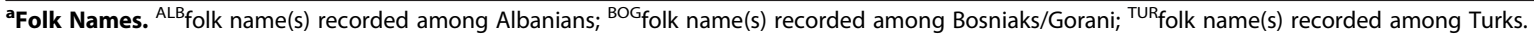

${ }^{\mathbf{b}} \mathbf{A l b} \mathbf{N}_{\mathbf{u c}}$ : Number of use citations provided by Albanian informants; Bo/Go $\mathbf{N}_{\mathbf{u c}}$ : Number of use citations provided by Bosnian and Gorani informants; Tur $\mathbf{N}_{\mathbf{u c}}$ : Number of use citations provided by Turkish informants.

'UV $_{\mathbf{A l b}}$ : Use-value for one species by the Albanian group; $\mathbf{U V}_{\mathbf{B o} / \mathbf{G o}}$ : Use-value for one species by the Bosniaks and Gorani; $\mathbf{U V}_{\mathbf{T u r}}$ : Use-value for one species by the Turkish group. This index measures the relative importance of each species based on its reported use by informants from each cultural group under study.

${ }^{d} \mathbf{Q}$ : Quadrant assignments are based on adjusted use-values $\left(U V_{\text {adj }}\right)$, which were calculated by dividing the use-value (UV) of each group by the maximum usevalue $\left(U V_{\text {max }}\right)$ for food citations (UV adj not shown).

- the topical application of Hordeum sativum flour, mixed with oil, for wound healing;

- the drinking of an infusion of the aerial parts of Juncus effusus (Figure 6) to treat urinary tract disorders;

- the drinking of an infusion of the aerial parts of Leonurus cardiaca as cardiotonic, to improve blood circulation and memory enhancement; and

- the drinking of an infusion of aerial parts of Trifolium arvense as an anti-rheumatic.

\section{Food plants}

The food uses of 29 wild species, representing 16 families, were recorded (Table 2). Of these, 3 were quoted only by Albanians, 2 only by Turks and 2 only by Bosniaks/ Gorani. Figure 3B illustrates the high level of overlap of cited plant species for food uses, with 12 species being cited by all three populations. Regarding common overlaps in species uses, 1were shared in common only between Albanians and Turks, 4 only between Bosniaks/ Gorani and Turks and 5 only between Albanian and Bosniaks/Gorani. Regarding the preparation of traditional foods, some of these, such as salep (beverage from Orchis spp. tubers) and shurup (syrup from Rosa damascena flowers), were prepared quite frequently in the past, but nowadays have nearly disappeared. The most frequently cited food uses of local plants referred to foods that are eaten fresh or processed (33.3\%), beverages $(22.2 \%)$, teas $(17.8 \%)$, jams $(17.8 \%)$ and food additives (8.9\%). Our 3-D analysis of adjusted use values revealed that all taxa with the exception of one are placed in Quadrant I, indicating a common low to moderate level of use-value shared among populations. Wild strawberries (Fragaria vesca), on the other hand, fell in Quadrant II, and is highly valued by Bosniaks/Gorani for its use as a food, beverage ingredient and jam ingredient. Its use as a beverage by the Bosniak/Gorani, prepared by boiling with sugar, was not cited by either Albanians or Turks in this study.

\section{Handicraft plants}

The handicraft uses of 20 species, representing 18 families, were recorded (Table 3 ). Of these, 5 were quoted only by Albanians, 3 by Turks and 2 by Bosniaks/Gorani. Figure 3C illustrates a moderate level of overlap of the handicraft uses of plant species, with only 4 being cited by all 3 populations. Regarding common overlaps in species uses, 3 were shared in common only between Albanians and Turks, 1 only between Bosniaks/Gorani and Turks and 2 only between Albanian and Bosniaks/Gorani. The most frequently cited form of handicraft uses of local flora included dyes (38.1\%), musical instruments (28.6\%), carpentry (19.0\%) and liquid containers (14.3\%).

As might be expected with lower levels of overlap between taxa cited for use for this purpose, we also observed greater distinction in the spread of taxa in our 3-D comparative analysis of adjusted use-values. Of note, Corylus avellana, which is a key resource for basket weaving in this region, fell into Quadrant VI, indicating its high use-value among Albanians and Bosniaks/ Gorani. It had no cited use among Turks. Lagenaria siceraria, whose fruits are used as a container for carrying water, had a top use-value among Bosniaks/Gorani, with moderate scores among Albanians and Turks (Quadrant II). Pinus nigra, used for home and furniture construction, likewise has a high use-value score among 
Table 3 Plants used in handicraft applications in the study area

\begin{tabular}{|c|c|c|c|c|c|c|c|c|c|c|c|c|}
\hline $\begin{array}{l}\text { Botanical taxon, } \\
\text { family and } \\
\text { voucher specimen } \\
\text { code }\end{array}$ & Status & $\begin{array}{l}\text { Folk } \\
\text { name(s) }\end{array}$ & $\begin{array}{l}\text { Part(s) } \\
\text { used }\end{array}$ & $\begin{array}{l}\text { Use } \\
\text { Category }\end{array}$ & Specific Use(s) & $\begin{array}{l}\text { Alb } \\
\mathrm{N}_{\mathrm{uc}}\end{array}$ & $\begin{array}{l}\text { Bo/ } \\
\mathrm{Go} \\
\mathrm{N}_{\mathrm{uc}}^{\mathrm{b}}\end{array}$ & $\begin{array}{l}\text { Tur } \\
\mathrm{N}_{\mathrm{uc}}^{\mathrm{b}}\end{array}$ & $\mathrm{UV}_{\mathrm{clb}}$ & $\begin{array}{l}\mathrm{UV}_{\mathrm{Bo}} / \\
\mathrm{Go}^{\mathrm{C}}\end{array}$ & UVVur & $Q^{d}$ \\
\hline $\begin{array}{l}\text { Abies alba Mill. } \\
\text { (Pinaceae) 14/ } \\
\text { Pz/2013 }\end{array}$ & W & Bredhi $i^{\mathrm{ALB}}$ & Wood & Carpentry & $\begin{array}{l}\text { Used for home construction and } \\
\text { different home furniture }\end{array}$ & 5 & 4 & 2 & 0.082 & 0.125 & 0.043 & I \\
\hline $\begin{array}{l}\text { Acer campestre L. } \\
\text { (Sapindaceae) }\end{array}$ & W & Panja ALB & Wood & Carpentry & $\begin{array}{l}\text { Used for constructing musical } \\
\text { instruments ("çifteli", violin etc.) }\end{array}$ & 2 & 0 & 0 & 0.033 & 0 & 0 & 1 \\
\hline $\begin{array}{l}\text { Alnus glutinosa L. } \\
\text { (Betulaceae) }\end{array}$ & W & Verri $^{A L B}$ & Twigs & Dye & $\begin{array}{l}\text { Brown color used for textile } \\
\text { coloring }\end{array}$ & 2 & 1 & 1 & 0.033 & 0.031 & 0.022 & I \\
\hline $\begin{array}{l}\text { Beta vulgaris L. } \\
\text { (Amaranthaceae) }\end{array}$ & C & Rrepa $a^{A L B}$ & Taproot & Dye & Red color, used for textile coloring & 2 & 0 & 0 & 0.033 & 0 & 0 & । \\
\hline \multirow{2}{*}{$\begin{array}{l}\text { Centaurea cyanus L. } \\
\text { (Asteraceae) 20/ } \\
\text { Pz/2013 }\end{array}$} & \multirow[t]{2}{*}{ W } & KokoçeliA $^{\text {ALB }}$ & \multirow[t]{2}{*}{ Flowers } & \multirow[t]{2}{*}{ Dye } & \multirow[t]{2}{*}{ Blue color, used for textile coloring } & \multirow[t]{2}{*}{0} & \multirow[t]{2}{*}{5} & \multirow[t]{2}{*}{0} & \multirow[t]{2}{*}{0} & \multirow[t]{2}{*}{0.156} & \multirow[t]{2}{*}{0} & $\|$ \\
\hline & & Kicica ${ }^{\mathrm{BOG}}$ & & & & & & & & & & \\
\hline $\begin{array}{l}\text { Corylus avellana L. } \\
\text { (Betulaceae) 24/ } \\
\text { Pz/2013 }\end{array}$ & W & Lejthı ${ }^{A L B}$ & Stems & Handicraft & $\begin{array}{l}\text { Used to construct baskets, usually } \\
\text { large ones for carrying animal food }\end{array}$ & 10 & 5 & 0 & 0.164 & 0.156 & 0 & $\mathrm{Vl}$ \\
\hline \multirow{3}{*}{$\begin{array}{l}\text { Cotinus coggygria } \\
\text { Scop. } \\
\text { (Anacardiaceae) 64/ } \\
\text { Pz/2013 }\end{array}$} & \multirow[t]{3}{*}{ W } & Dru boje ${ }^{A L B}$ & \multirow[t]{3}{*}{ Fruits } & \multirow[t]{3}{*}{ Dye } & \multirow{3}{*}{$\begin{array}{l}\text { Yellow color, used for leather, } \\
\text { wool and other textile coloring }\end{array}$} & \multirow[t]{3}{*}{2} & \multirow[t]{3}{*}{0} & \multirow[t]{3}{*}{3} & \multirow[t]{3}{*}{0.033} & \multirow[t]{3}{*}{0} & \multirow[t]{3}{*}{0.065} & । \\
\hline & & Ruj $^{\mathrm{TU}}$ & & & & & & & & & & \\
\hline & & $\begin{array}{l}\text { Boyaci } \\
\text { sumak }^{\text {TUR }}\end{array}$ & & & & & & & & & & \\
\hline \multirow[t]{2}{*}{$\begin{array}{l}\text { Juglans regia L. } \\
\text { (Juglandaceae) 40/ } \\
\text { Pz/2013 }\end{array}$} & \multirow[t]{2}{*}{ C } & \multirow[t]{2}{*}{ Arra ${ }^{A L B}$} & Wood & Carpentry & $\begin{array}{l}\text { Used for furniture preservation, this } \\
\text { is characterized by a high aesthetic } \\
\text { value. }\end{array}$ & 3 & 2 & 2 & 0.082 & 0.063 & 0.043 & । \\
\hline & & & $\begin{array}{l}\text { Fruit } \\
\text { cortex }\end{array}$ & Dye & Coloring of hair, wool and cotton & 2 & 0 & 0 & & & & \\
\hline $\begin{array}{l}\text { Juniperus communis } \\
\text { L. (Cupressaceae) } \\
\text { 39/Pz/2013 }\end{array}$ & W & Gëllija ${ }^{A L B}$ & Wood & $\begin{array}{l}\text { Musical } \\
\text { instrument }\end{array}$ & $\begin{array}{l}\text { Used for construction of "lahuta", a } \\
\text { single-stringed musical instrument } \\
\text { used in traditionally music. }\end{array}$ & 2 & 0 & 0 & 0.033 & 0 & 0 & । \\
\hline $\begin{array}{l}\text { Lagenaria siceraria } \\
\text { (Molina) Standl. } \\
\text { (Cucurbitaceae) }\end{array}$ & C & Pocerka ${ }^{A L B}$ & $\begin{array}{l}\text { Dried } \\
\text { fruits }\end{array}$ & $\begin{array}{l}\text { Liquid } \\
\text { container }\end{array}$ & $\begin{array}{l}\text { Fruits opened and used as a water } \\
\text { container }\end{array}$ & 6 & 8 & 4 & 0.098 & 0.25 & 0.087 & $\|$ \\
\hline \multirow{2}{*}{$\begin{array}{l}\text { Morus alba L. } \\
\text { (Moraceae) 49/ } \\
\text { Pz/2013 }\end{array}$} & \multirow[t]{2}{*}{ C } & $\begin{array}{l}\text { Mani i } \\
\text { bardhëelB }\end{array}$ & \multirow[t]{2}{*}{ Wood } & $\begin{array}{l}\text { Liquid } \\
\text { container }\end{array}$ & $\begin{array}{l}\text { Used to construct casks for storing } \\
\text { alcohol, which gives it a }\end{array}$ & 4 & 0 & 1 & 0.066 & 0 & 0.022 & I \\
\hline & & Akdut $^{\text {TUR }}$ & & & & & & & & & & \\
\hline $\begin{array}{l}\text { Morus nigra L. } \\
\text { (Moraceae) 48/ }\end{array}$ & C & $\begin{array}{l}\text { Mani i ziALB } \\
\text { Dut }^{\text {TUR }}\end{array}$ & Wood & $\begin{array}{l}\text { Liquid } \\
\text { container }\end{array}$ & $\begin{array}{l}\text { Used to construct casks for storing } \\
\text { alcohol, which gives it a }\end{array}$ & 4 & 0 & 1 & 0.066 & 0 & 0.022 & । \\
\hline & & Karadut $^{\text {TUR }}$ & & & & & & & & & & \\
\hline Pinus nigra J.F. & W/C & Pisha ${ }^{A L B}$ & Wood & Carpentry & Used for home construction and & 0 & 6 & 1 & 0 & 0.188 & 0.022 & $\|$ \\
\hline & & Kara qam ${ }^{\text {TUR }}$ & & & & & & & & & & \\
\hline Polygonum aviculare & W & Madimak $^{\text {BOG }}$ & Aerial & Dye & Blue color, used for wool coloring & 0 & 0 & 3 & 0 & 0 & 0.065 & । \\
\hline & & Kusekmezi ${ }^{\text {TuR }}$ & & & & & & & & & & \\
\hline Pyrus communis L. & W & Dardha ${ }^{A L B}$ & Wood & Musical & Used for construction of "Zurla", an & 2 & 0 & 0 & 0.033 & 0 & 0 & । \\
\hline $\begin{array}{l}\text { (Rosaceae) 58/ } \\
\mathrm{Pz} / 2013\end{array}$ & & $\begin{array}{l}\text { Dardha } \\
\text { eger }^{A L B}\end{array}$ & & & & & & & & & & \\
\hline $\begin{array}{l}\text { Rhamnus frangula } \\
\text { (Rhamnaceae) }\end{array}$ & $\mathrm{E}$ & $\begin{array}{l}\text { Druni } \\
\text { barutit }^{\mathrm{ALB}}\end{array}$ & Wood & Weaponry & Used as a gunpowder ingredient & 0 & 0 & 1 & 0 & 0 & 0.022 & । \\
\hline & & $\begin{array}{l}\text { Barut } \\
\text { agaqi }\end{array}$ & & & & & & & & & & \\
\hline $\begin{array}{l}\text { Rubia tinctorum L. } \\
\text { (Rubiaceae) }\end{array}$ & W & $\begin{array}{l}\text { Boj kuqe }{ }^{\text {ALB }} \\
\text { Crvenka }^{\text {BOG }}\end{array}$ & $\begin{array}{l}\text { Roots } \\
\text { and } \\
\text { fruits }\end{array}$ & Dye & Red color, used for textile coloring & 0 & 4 & 0 & 0 & 0.125 & 0 & । \\
\hline & W & Rakita ${ }^{\mathrm{ALB}}$ & Twigs & Handicraft & & 5 & 2 & 0 & 0.082 & 0.063 & 0 & I \\
\hline
\end{tabular}


Table 3 Plants used in handicraft applications in the study area (Continued)

\begin{tabular}{|c|c|c|c|c|c|c|c|c|c|c|c|c|}
\hline $\begin{array}{l}\text { Salix purpurea L. } \\
\text { (Salicaceae) }\end{array}$ & & & & & $\begin{array}{l}\text { To construct different type of } \\
\text { baskets }\end{array}$ & & & & & & & \\
\hline \multirow{2}{*}{$\begin{array}{l}\text { Sambucus ebulus L. } \\
\text { (Adoxaceae) }\end{array}$} & \multirow[t]{2}{*}{ W } & Kinla ${ }^{A L B}$ & \multirow[t]{2}{*}{ Fruits } & \multirow[t]{2}{*}{ Dye } & \multirow[t]{2}{*}{ Blue color, used for textile coloring } & \multirow[t]{2}{*}{0} & \multirow[t]{2}{*}{4} & \multirow[t]{2}{*}{0} & \multirow[t]{2}{*}{0} & \multirow[t]{2}{*}{0.125} & \multirow[t]{2}{*}{0} & । \\
\hline & & Crna zova ${ }^{\mathrm{BOG}}$ & & & & & & & & & & \\
\hline \multirow{3}{*}{$\begin{array}{l}\text { Zea mays L. } \\
\text { (Poaceae) 92/Pz/ } \\
2013\end{array}$} & \multirow[t]{3}{*}{ C } & Misri ${ }^{A L B}$ & \multirow{3}{*}{$\begin{array}{l}\text { Mature } \\
\text { leaves }\end{array}$} & \multirow[t]{3}{*}{ Handicraft } & \multirow{3}{*}{$\begin{array}{l}\text { Used to construct different types } \\
\text { of baskets }\end{array}$} & \multirow[t]{3}{*}{0} & \multirow[t]{3}{*}{0} & \multirow[t]{3}{*}{4} & \multirow[t]{3}{*}{0} & \multirow[t]{3}{*}{0} & \multirow[t]{3}{*}{0.087} & I \\
\hline & & Kollomoq ${ }^{\text {ALB }}$ & & & & & & & & & & \\
\hline & & Kollomoqi $^{\text {TUR }}$ & & & & & & & & & & \\
\hline
\end{tabular}

${ }^{\text {a }}$ Folk Names. ${ }^{\text {ALB }}$ folk name(s) recorded among Albanians; ${ }^{B_{0}}$ folk name(s) recorded among Bosniaks/Gorani; ${ }^{\text {TUR }}$ folk name(s) recorded among Turks.

${ }^{\mathbf{b}}$ Alb $\mathbf{N}_{\mathbf{u c}}$ : Number of use citations provided by Albanian informants; Bo/Go $\mathbf{N}_{\mathbf{u c}}$ : Number of use citations provided by Bosnian and Gorani informants; Tur $\mathbf{N}_{\mathbf{u c}}$ : Number of use citations provided by Turkish informants.

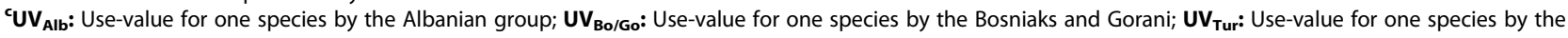
Turkish group. This index measures the relative importance of each species based on its reported use by informants from each cultural group under study. ${ }^{d} Q$ : Quadrant assignments are based on adjusted use-values $\left(U_{\text {adj }}\right)$, which were calculated by dividing the use-value (UV) of each group by the maximum use-value $\left(U V_{\max }\right)$ for handicraft citations (UV adj not shown).

Bosniaks/Gorani, but a very low use-value among Turks, and no citations for Albanians.

\section{Cross-cultural comparison}

Both the distinct and overlapping patterns of TEK reported by the 3 ethnic groups are illustrated in Figure 3. Although the number of informants was slightly uneven among the three populations, a general tendency can be observed nevertheless, also because "saturation" plateaus in which no new plant uses quoted by new interviewees were commonly reached after approximately 15-20 interviews. While we could not observe any remarkable differences among the wild plants used in the food and handicraft domains by the three populations, a difference is notable in the medicinal domain. When it comes to medicinal TEK, Albanians appear less herbophilic than both Slavs and Turks. This finding confirms what has already been pointed out by other field studies conducted in other Western Balkans areas and involving both Slavs and Ghegh Albanians [10,15]. This phenomenon may be best explained by the fact that the traditional

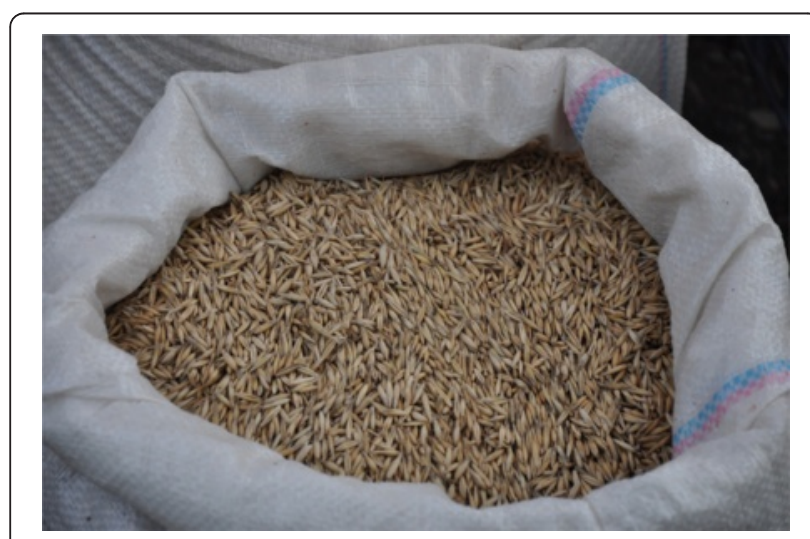

Figure 5 The aerial parts of Avena sativa L. (Poaceae) are prepared as an infusion and drunk for the purpose of enhancing the skeletal system. economy of Ghegh Albanians was for many centuries based upon a pure pastoralist/transhumant economy, whereas they have rarely traded herbs. For the Slavs, however, the gathering of herbs from the wild has persisted as their well-known main occupation within a mixed system of small-scale agriculture and pastoralism. This is especially the case among Islamicized Slavs living in the mountainous areas of SE Europe.

\section{Conclusion}

For the first time in European ethnobotany, this study presents data comparing the medicinal, food, and handicraft plant use practices of three different ethnic populations living in the same area. We have introduced a new analytical method (3-D adjusted use-value plots) for comparison of taxa across different populations living in the same environment, with access to the same taxa and other environmental resources. While we have documented the presence of some small distinct sets of TEK in these populations, this is overwhelmingly coupled by a substantial overlap in the use of local taxa, suggesting

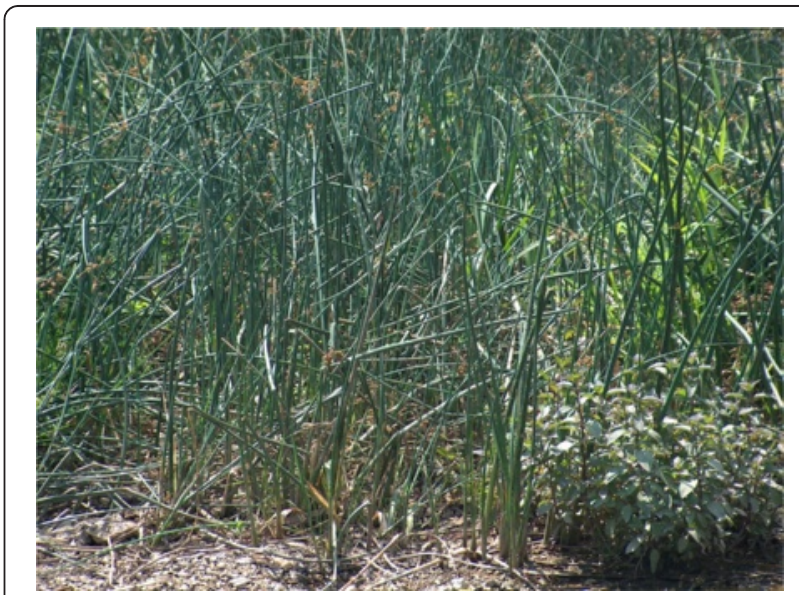

Figure $\mathbf{6}$ The aerial parts of Juncus effusus L. (Juncaceae) are prepared as an infusion and drunk to treat urinary tract disorders. 
a hybrid character to the Kosovar TEK in this region, especially with regards to TEK in the food and handicraft domains. Such cross-cultural studies could be important for proposing culturally-sensitive ways of using plant natural resources in future sustainable economic development initiatives. Indeed, the success of any future development efforts involving natural resources must take into account local perceptions and attitudes concerning plants, which can vary greatly in some cases, among different ethnic groups living in the same territory. Examples of such initiatives could include a focus on eco-tourism and the small-scale trade of foods, aromatic plants, medicinal herbs, and handicraft products. Findings from studies such as this one should be implemented in projects aimed at fostering collaboration and reconciliation among the diverse ethnic and religious communities living in Kosovo.

\section{Competing interest}

The authors declare that they have no competing interest.

\section{Authors' contributions}

$\mathrm{BM}$ and $\mathrm{AH}$ conceived and designed the study, XK, BP, AH performed the interviews, and $C Q, A P$ and $A H$ analyzed the data. $A H$ and $B M$ wrote the paper; AP and CQ provided revisions. All authors read and approved the final manuscript.

\section{Acknowledgments}

We would like to extend our heartfelt thanks to the communities and people who agreed to participate in this study; moreover, we thank the US Embassy in Kosovo, for financial support of the field research (grant no. S-KV420-14GR-096). Thanks to Matthew Dorian for assistance with MATLAB ${ }^{\oplus}$ programing.

\section{Author details \\ ${ }^{1}$ Institute of Biological and Environmental Research, University of Prishtina "Hasan Prishtina", Mother Teresa, 1000 Prishtinë, Kosovo. ${ }^{2}$ University of Gastronomic Sciences, Piazza Vittorio Emanuele 9, I-12060 Pollenzo, Italy. ${ }^{3}$ Center for the Study of Human Health, Emory University, 550 Asbury Circle, Candler Library 107E, Atlanta, GA 30322, USA. ${ }^{4}$ Department of Dermatology, Emory University School of Medicine, 1518 Clifton Rd NE, CNR Bldg. 5000, Atlanta, GA 30322, USA.}

Received: 20 January 2015 Accepted: 22 April 2015

Published online: 12 May 2015

\section{References}

1. Šarić-Kundalić B, Dobeš C, Klatte-Asselmeyer V, Saukel J. Ethnobotanical survey of traditionally used plants in human therapy of east, north and north-east Bosnia and Herzegovina. J Ethnopharmacol. 2011;133(3):1051-76.

2. Šarić-Kundalić B, Dobeš C, Klatte-Asselmeyer V, Saukel J. Ethnobotanical study on medicinal use of wild and cultivated plants in middle, south and west Bosnia and Herzegovina. J Ethnopharmacol. 2010;131(1):33-55.

3. Šarić-Kundalić B, Fritz E, Dobeš C, Saukel J. Traditional medicine in the pristine village of Prokoško lake on Vranica Mountain, Bosnia and Herzegovina. Sci Pharm. 2010;78(2):275-90.

4. Redžić $\mathrm{S}$. Wild medicinal plants and their usage in traditional human therapy (Southern Bosnia and Herzegovina, W. Balkan). J Med Plant Res. 2010;4(11):1003-27.

5. Redžić S. Wild Mushrooms and Lichens used as Human Food for Survival in War Conditions; Podrinje - Zepa Region (Bosnia and Herzegovina, W. Balkan). Res. Hum Ecol. 2010;17(2):175-87.

6. Redžic S. The ecological aspect of ethnobotany and ethnopharmacology of population in Bosnia and Herzegovina. Collegium Antropol. 2007;31(3):869-90.
7. Redžić $\mathrm{S}$. Wild edible plants and their traditional use in the human nutrition in Bosnia and Herzegovina. Ecol Food Nutr. 2006;45(3):189-232.

8. Zlatković BK, Bogosavljević SS, Radivojević AR, Pavlović MA. Traditional use of the native medicinal plant resource of Mt. Rtanj (Eastern Serbia): Ethnobotanical evaluation and comparison. J Ethnopharmacol. 2014;151(1):704-13.

9. Savikin K, Zdunic G, Menkovic N, Zivkovic J, Cujic N, Terescenko M, et al. Ethnobotanical study on traditional use of medicinal plants in SouthWestern Serbia, Zlatibor district. J Ethnopharmacol. 2013;146(3):803-10.

10. Pieroni A, Giusti ME, Quave CL. Cross-Cultural Ethnobiology in the Western Balkans: Medical Ethnobotany and Ethnozoology Among Albanians and Serbs in the Pešter Plateau, Sandžak, South-Western Serbia. Hum Ecol. 2011;39(3):333-49.

11. Tomićević J, Bjedov I, Obratov-Petković D, Milovanović M. Exploring the park-people relation: Collection of Vaccinium myrtillus $L$. by local people from Kopaonik National Park in Serbia. Environ Manage. 2011;48(4):835-46.

12. Jarić S, Popović Z, Mačukanović-Jocić M, Djurdjević L, Mijatović M, Karadžić $B$, et al. An ethnobotanical study on the usage of wild medicinal herbs from Kopaonik Mountain (Central Serbia). J Ethnopharmacol. 2007;111(1):160-75.

13. Menković N, Šavikin K, Tasić S, Zdunić G, Stešević D, Milosavljević S, et al. Ethnobotanical study on traditional uses of wild medicinal plants in Prokletije Mountains (Montenegro). J Ethnopharmacol. 2011;133(1):97-107.

14. Pieroni A, Nedelcheva A, Hajdari A, Mustafa B, Scaltriti B, Cianfaglione K, et al. Local knowledge on plants and domestic remedies in the mountain villages of Peshkopia (Eastern Albania). J Mt Sci. 2014;11(1):180-94.

15. Pieroni AC K, Nedelcheva A, Hajdari A, Mustafa B, Quave CL. Resilience at the border: traditional botanical knowledge among Macedonians and Albanians living in Gollobordo, Eastern Albania. J Ethnobiol Ethnomed. 2014;31:10.

16. Quave CL, Pieroni A. Fermented foods for food security and food sovereignty in the Balkans: A case study of the Gorani people of Northeastern Albania. J Ethnobiol. 2014;34(1):28-43.

17. Pieroni A. People and plants in Lëpushë. Traditional medicine, local foods, and post- communism in a North Albanian village. In: PardodeSantayana M, Pieroni A, Puri R, editors. Ethnobotany in the new Europe: People, Health and Wild Plant Resources. New York/Oxford: Berghahn; 2010. p. 16-50.

18. Pieroni A. Local plant resources in the ethnobotany of Theth, a village in the Northern Albanian Alps. Genet Resour Crop Ev. 2008;55(8):1197-214.

19. Pieroni A, Dibra B, Grishaj G, Grishaj I, Maçai SG. Traditional phytotherapy of the Albanians of Lepushe, Northern Albanian Alps. Fitoterapia. 2005;76(3-4):379-99.

20. Pieroni A, Rexhepi B, Nedelcheva A, Mustafa B, Hajdari A, Kolosova V, et al. One century later: the folk botanical knowledge of the last remaining Albanians of the upper Reka Valley, Mount Korab, Western Macedonia. J Ethnobiol Ethnomed. 2013;9:22

21. Rexhepi B, Mustafa B, Hajdari A, Rushidi-Rexhepi J, Quave CL, Pieroni A. Traditional medicinal plant knowledge among Albanians, Macedonians and Gorani in the Sharr Mountains (Republic of Macedonia). Genet Resour Crop Ev. 2013:60(4):2055-80

22. Lescureux N, Linnell JDC, Mustafa S, Melovski D, Stojanov A, Ivanov G, et al. Fear of the unknown: Local knowledge and perceptions of the Eurasian lynx Lynx lynx in western Macedonia. ORYX. 2011;45(4):600-7.

23. Lescureux N, Linnell JDC, Mustafa S, Melovski D, Stojanov A, Ivanov G, et al. The king of the forest: Local knowledge about European brown bears (Ursus arctos) and implications for their conservation in contemporary Western Macedonia. Conserv Soc. 2011;9(3):189-201.

24. Lescureux N, Linnell JDC. Knowledge and perceptions of Macedonian hunters and herders: The influence of species specific ecology of bears, wolves, and lynx. Hum Ecol. 2010;38(3):389-99.

25. Mustafa B, Hajdari A, Krasniqi F, Hoxha E, Ademi H, Quave CL, et al. Medical ethnobotany of the Albanian Alps in Kosovo. J Ethnobiol Ethnomed. 2012;8:6.

26. Mustafa B, Hajdari A, Pajazita Q, Syla B, Quave CL, Pieroni A. An ethnobotanical survey of the Gollak region, Kosovo. Genet Resour Crop Ev. 2011;59(5):739-54.

27. Sejdiu S. Fjalorth ethnobotanik i shaipes. Rilindja: Prishtina; 1984.

28. Cukić D. Turistička valorizacija prirodnih, etnografskih i drugih kulturnih potencijala Šar-planine. Belgrade: Srpsko Geografsko Društvo; 1983.

29. Krasniqi F. Promene u flori i vegetacije Kosova poslednjih decenija i mere njihove zashtite. Makedonska Akademija na Naukite i Umetnostite. 1982;ll:59-67. 
30. Rexhepi F. Vegjetacioni i Kosovës. Prishtina: FSHMN, Universiteti i Prishtinë; 1994.

31. Mustafa B, Hajdari A, Veselaj Z, Beadini B, Ibrahimi H, Mustafa N, et al. Extension of the National Park "Sharri" boundaries, significant action for preservation of natural values. Natura Montenegrina. 2013;12(1):607-16.

32. Schmitt O. Kosovo: kurze Geschichte einer zentralbalkanischen Landschaft. Vienna: Böhlau; 2008

33. Kosovo Agency of Statistics: http://ask.rks-gov.net/publikimet/ cat view/8-popullsia

34. The International Society of Ethnobiology Code of Ethics. 2006.http:// ethnobiology.net/code-of-ethics/. Accessed 18 January 2015.

35. Paparisto K, Vangjeli J, Ruci B, Mullaj A, Qosja X: Flora e Shqipërisë. Vol. 1-4; Tirana: ASHASH, Instituti i Kërkimeve Biologjike; 1988-2000.

36. Jordanov D: Flora NR Bulgaria I-VII; Sofia: BANU; 1963-1979.

37. Pajazitaj Q. Përcaktuesi i bimëve Pteridofite dhe Spermatofite. Prishtina: Universiteti i Prishtinës; 2004

38. Demiri M. Flora ekskursioniste e Shqipërisë. Tirana: Libri Shkollor; 1981

39. Tutin T, Heywood V, Burges N, Valentine D, Walters S, Webb D. Flora Europaea. Cambridge, UK: University Press; 1964.

40. Stevens P. Angiosperm Phylogeny Website. Version 12. 2012. http:// www.mobot.org/MOBOT/research/APweb/. Accessed 18 January 2015.

41. Albuquerque UP, Medeiros PM, Almeida AL, Monteiro JM, Lins Neto EMF Melo JG, et al. Medicinal plants of the caatinga (semi-arid) vegetation of NE Brazil: a quantitative approach. J Ethnopharmacol. 2007;114:325-54.

42. Quave $\mathrm{CL}$, Pieroni A: A reservoir of ethnobotanical knowledge informs resilient food security and health strategies in the Balkans. Nature Plants. 2015. DOI: 10.1038/NPLANTS.2014.21.

\section{Submit your next manuscript to BioMed Central and take full advantage of:}

- Convenient online submission

- Thorough peer review

- No space constraints or color figure charges

- Immediate publication on acceptance

- Inclusion in PubMed, CAS, Scopus and Google Scholar

- Research which is freely available for redistribution 